\title{
Quantum interference and the formation of the proximity effect in chaotic normal-metal/superconducting structures
}

\author{
D. Taras-Semchuk and Alexander Altland \\ Theoretische Physik III, Ruhr-Universität-Bochum, 44780 Bochum, Germany
}

\begin{abstract}
We discuss a number of basic physical mechanisms relevant to the formation of the proximity effect in superconductor/normal metal (SN) systems. The physics of this effect, most notably the phenomenon of density of states depletion in normal metals adjacent to superconductors, sensitively depends on various aspects of quantum interference and chaotic charge carrier dynamics in mesoscopic systems. Specifically, we review why the proximity effect sharply discriminates between systems with integrable and chaotic dynamics, respectively, and how this feature can be incorporated into theories of SN systems. Turning to less well investigated terrain, we discuss the impact of quantum diffractive scattering on the structure of the density of states in the normal region. We consider ballistic systems weakly disordered by pointlike impurities as a test case and demonstrate that diffractive processes akin to normal metal weak localization lead to the formation of a hard spectral gap - a hallmark of SN systems with chaotic dynamics. Turning to the more difficult case of clean systems with chaotic boundary scattering, we argue that semiclassical approaches, based on classifications in terms of classical trajectories, cannot explain the gap phenomenon. Employing an alternative formalism based on elements of quasiclassics and the ballistic $\sigma$-model, we demonstrate that the inverse of the so-called Ehrenfest time is the relevant energy scale in this context. We discuss some fundamental difficulties related to the formulation of low energy theories of mesoscopic chaotic systems in general and how they prevent us from analysing the gap structure in a rigorous manner. Given these difficulties, we argue that the proximity effect represents a basic and challenging test phenomenon for theories of quantum chaotic systems.
\end{abstract}

PACS numbers: 73.23.Ad,73.20.Fz,05.45.Mt,74.50.+r

\section{INTRODUCTION}

Superconductors attached to normal metals tend to export aspects of their anomalous properties into the adjacent normal metal region. Termed the proximity effect, this tendency manifests itself in phenomena such as the Josephson effects, anomalous charge and thermal transport coefficients, and altered thermodynamic properties (see e.g. Refs. [1] [3] for extensive reviews). All these effects find their common origin in the fact that a superconductor pairing field amplitude may penetrate deeply into the normal metal region, before decaying due to the absence of attractive interaction mechanisms.

Perhaps the most direct manifestation of the presence of a finite pairing field amplitude in a normal metal adjacent to a superconductor is a massive suppression of the single particle density of states (DoS). This phenomenon, first noticed almost forty years ago, has a long history of research. Beginning with a classic paper of de Gennes

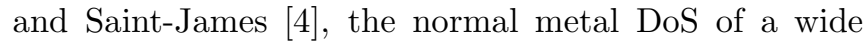
family of superconductor-normal-metal hybrid systems (SN-systems) has been investigated. Among these are superconductors coated with normal metal thin films, both clean and disordered [4.5], bulk diffusive compounds in contact with superconductor terminals [6 10] and various types of SN quantum dot structures [1,11 [16].

In general the quantitative functional form of the energy dependent DoS, $\nu(\epsilon)$, of any of these systems depends on the system category, and on a number of relevant material parameters. Interestingly, however, a simple ordering principle exists, as first pointed out by Melsen et al. [12,13], according to which the qualitative structure of the DoS falls into one of only two different categories: For systems whose normal compound is integrable - in the sense that the dynamics of the single particle degrees of freedom becomes integrable in the classical limit - the DoS vanishes in a close to linear fashion [4, 12, 13] as the excitation energy $\epsilon$ approaches zero. Of course, due to the inevitable presence of imperfections, truly integrable systems in condensed matter physics do not exist. However, a number of systems, e.g. thin clean metallic films, come reasonably close to the integrable limit implying that the proximity DoS obeys a continuously decreasing behavior down to very small excitation energies $\epsilon$. In contrast, the DoS of genuinely chaotic systems - significantly disordered systems or chaotic quantum dot structures - is gapped: $\nu(\epsilon)$ falls to zero at some finite threshold energy $\epsilon^{*}$. For energy values $|\epsilon|$ smaller than $\epsilon^{*}$, the DoS is strictly vanishing.

Beyond its practical relevance in the physics of SN systems, this twofold categorization scheme is of noticeable conceptual interest. Indeed, the observation that a quantity as elementary as the single particle DoS of may serve as an indicator sharply discriminating between integrable and chaotic dynamics has led to a wave of interest in the interplay between superconductor correlations and quantum chaotic dynamics 11 13, 17 21]. Unfortunately, the established tools of theoretical mesoscopic superconductivity - quasiclassical Green function methods and diagrammatic techniques - are not straightforwardly appli- 
cable to clean chaotic SN structures. As an alternative to direct numerical analyses, an approach based on classical path counting, augmented by a Bohr-Sommerfeld quantization rule, has become popular. Referring to a more substantial discussion to below, we here merely note that the Bohr-Sommerfeld (BS) approach readily produces a massive suppression of the low energy DoS of chaotic systems [17,18 (as compared to the integrable case.) Furthermore, the energetic extension of the depletion region turns out to be in good accord with the results obtained by direct diagonalization 12,13,17, 18, 21. In this sense, the BS quantization scheme represents a comparatively simple and widely applicable tool for exploring the DoS of SN systems.

There is, however, one key feature of the DoS gap that is missed by the BS-scheme: for chaotic systems the gap tends to be hard (in the sense that $\nu(\epsilon)$ strictly vanishes for energies $|\epsilon|<\epsilon^{*}$.) Hard gaps are found for a wide class of chaotic SN systems: For the particular case of diffusive SN systems, the structure of a hard gap has been obtained analytically by quasiclassical methods [22,6 10 . A numerical study by Pilgram et al. [23] has shown that the gap of weakly disordered systems, i.e. systems whose spatial extent is smaller than the elastic mean free path, is also hard (within, of course, the numerical accuracy). The same applies to the numerically obtained DoS profiles of systems with smooth chaotic boundary scattering displayed in Ref. [17]. Finally, chaotic systems that are completely ergodic, in the sense that their quantum mechanics can be modelled by random matrix theory, also exhibit a hard gap [12,13].

In contrast, the very construction of the BS scheme implies that it categorically produces a finite (albeit small) DoS at non-zero energies [12,13, 17, 18,21]. That no gap is seen has to do with the fact that the the BS approach does not fully account for the quantum mechanical nature of the quasi-particle propagation in an SN environment. More specifically, the elimination of all states below a certain threshold energy is caused by mechanisms of quantum diffraction similar to those responsible for weak localization corrections in normal metals. The essential difference to the weak localization processes operative in normal mesoscopic systems is that diffraction processes in SN systems are not small. Indeed it is one of the main objectives of the present paper to show that at low enough energies these 'corrections' diverge and that a non-perturbative treatment is needed to correctly obtain the gapped DoS.

Qualitatively, the results of our analysis may be summarized as follows: in chaotic SN systems, there are at least two different time scales that are of relevance for the low energy DoS. The first is the average classical time $t_{\mathrm{c}}$ it takes to establish contact with the superconductor. This scale can be set by be the diffusion time time through an extended disordered system, the inverse tunneling rate through a nearly insulating superconductor normal metal interface, or the average ballistic time of flight through a clean quantum dot. The second time scale is, in general, of quantum mechanical nature, and is the time $t_{\mathrm{d}}$ a minimal wave package takes to lose memory of its classical initial conditions. In a medium with hard ( $s$-wave) scatterers, $t_{\mathrm{d}}$ will coincide with the classical scattering time $\tau$. In systems with smooth scattering potentials - no matter whether disordered or clean $-t_{\mathrm{d}}$ is determined by the Ehrenfest time $t_{\mathrm{E}}$ [24], that is to be discussed extensively below. For the structure of the low energy DoS, the larger of these two timescales is of foremost relevance. For example, in a typical diffusive system with hard impurities, the scattering time is much shorter than both the diffusion time and the tunneling time into the superconductor. In this case, $t_{\mathrm{c}}>t_{\mathrm{d}}$ and the parametric scale determining the gap edge [6,8] 10$]$ is $t_{\mathrm{c}}^{-1}$.

In this paper the focus will be on the complementary class of systems with $t_{\mathrm{d}} \gg t_{\mathrm{c}}$. We will begin by considering the prototypical class of ballistic systems weakly polluted by some $s$-wave impurities and well coupled to a superconductor. For such systems the scattering time is larger than the ballistic time of flight (and the tunneling time to the superconductor) and the above condition is met. Operating within a synthesis of the quasiclassical formalism and the ballistic non-linear $\sigma$-model [25,26], we will identify the relevant quantum diffraction processes, and show how these contributions lead to a vanishing of the DoS for energies $\epsilon<\epsilon^{*} \sim \hbar /(2 \tau)$.

Building on the insight gained in this analysis, we will then proceed to the more complex problem of clean chaotic systems with smooth potentials. In this case, the relevant corrections to the classical picture emerge from an interplay of quantum wave packet spreading and the exponential trajectory divergence inherent to chaotic dynamics. The characteristic time scale for these processes is the aforementioned Ehrenfest time. Below we will demonstrate that the Ehrenfest time is the relevant energy scale for the DoS of chaotic systems with smooth scattering. It should be mentioned right away that the quantitative parts of this part of the analysis will include certain assumptions and, therefore, do not attain the status of a rigorous calculation. Further, our formalism is not capable of exploring structures in the close vicinity of the estimated gap edge $\sim \hbar / t_{\mathrm{E}}$. Notwithstanding these deficiencies, our analysis demonstrates the suppression of the DoS below its semiclassically estimated value. Although we cannot mathematically prove that this quantum suppression mechanism produces a hard gap, the close structural analogy with the hard scattering problem makes us believe that this is the case. That a hard gap of the order of the inverse Ehrenfest time should be generated has already been conjectured by Lodder and Nazarov [17.

We would like to point out that the phrase 'hard gap', as used in this paper, needs some qualification: refined analysis of the spectral structure of diffusive Andreev systems has shown the existence of a tail below the quasiclassical gap edge 38] that is exponentially small in $g$, where $g \gg 1$ is the dimensionless conductance of the 
normal component of the system. Similar contributions have been indentified in billiards with randomly fluctuating geometry [31]. In diffusive systems, the formation of these anomalous 'tail' states is due to exponentially rare potential configurations which lead to an effective decoupling of the superconductor and normal metal [10. An interesting question, not addressed in the present paper, is whether similar states exist in clean chaotic environments. It should be emphasized, though, that tail state formation is a mechanism conceptually independent from the quantum diffractive suppression of the semiclassical results discussed in this paper.

Finally, let us mention that much of the conceptual ground on which our analysis of the DoS in chaotic systems with smooth boundaries is based was laid in a seminal paper by Aleiner and Larkin 24] (AL). In this work the fundamental importance of the Ehrenfest time for the formation of weak localization corrections in normal chaotic systems was realized and substantiated. Many of the ideas first formulated by AL have been included in our analysis of the superconductor problem.

The paper is organized as follows. In section II, we discuss some general physics associated with Andreev reflection in SN structures, introduce the BS approach and discuss the role of quantum diffraction processes in establishing the gap in the spectrum of the normal region. In section III we discuss how the BS approach may be recovered from the quasiclassical Eilenberger equation in the diffractionless limit, and establish a simple trial solution of the quasiclassical equations in the presence of a collision operator. In section IV we introduce a functional integral formulation for the disordered Green function of an SN system in terms of a ballistic sigma-model. In section $\mathrm{V}$ we show how this formalism may be applied to determine the spectrum of a SN system with bulk, ballistic $s$-wave disorder, and establish the presence of a hard gap of the order of the inverse scattering time. In section VI we turn to the study of the DoS in clean chaotic SN systems, in the presence of a smooth scattering potential, and discuss the appearance of the inverse Ehrenfest time in the structure of the DoS. Section VII concludes with a discussion and summary.

\section{QUALITATIVE PHYSICS OF THE GAP}

The basic microscopic mechanism of coupling between a superconductor and an adjacent normal metal is Andreev reflection [27]: a normal metal electron impingent on the superconductor interface is converted into a hole (charge balance being restored by the formation of a superconductor Cooper pair) which is reflected back into the normal metal. This type of scattering has a number of peculiar properties which are at the root of the formation of the DoS gap and which are summarized below for later reference:

- Andreev reflection is a retro-reflection, i.e. the hole is essentially reflected back into the path of the incident electron where

- there is a slight angular mismatch set by the excitation energy $\epsilon$ of the electron above the Fermi energy $E_{F}$.

- For an electron of energy $\epsilon$, the reflected hole has energy $-\epsilon$.

- the hole acquires a scattering phase, which takes the value of $\pi / 2-\phi$ (for an excitation energy $\epsilon$ far below the superconducting gap), where $\phi$ is the value of the superconducting order parameter at the point of incidence.

From these properties, the origin of the DoS suppression in normal metals adjacent to superconductors may be readily understood. Assume that an electron of some small excitation energy $\epsilon$ is injected somewhere into the normal metal (at the beginning of the solid line in Fig. 1 say.) The electron will travel along some path $\gamma$ in the normal metal and at some point hit the superconductor where it is converted into a hole. The hole then propagates approximately (up to the above mentioned angular mismatch) back along the path $\gamma$ (dotted line in Fig. 11) and eventually arrives in the vicinity of the point of injection. Seen as a whole, this process amounts to the formation of a local net pair amplitude $\sim\left\langle\psi^{\dagger}(\epsilon) \psi^{\dagger}(\epsilon)\right\rangle$, i.e. an amplitude for the creation of an electron and an annihilation of a hole, in the normal metal. Importantly, this induced Cooper pair amplitude is not weighted by rapidly fluctuating quantum mechanical phases. To understand this point, notice that the quantum phase of the incident electron is essentially given by the classical action $S_{\gamma}(\epsilon)$ for traversal along $\gamma$. The action of the Andreev reflected hole (a missing electron) is given by $-S_{\gamma}(-\epsilon)$. These two contributions largely cancel against each other, implying that a slowly fluctuating net amplitude obtains.

Unitarity implies that the formation of a non-vanishing Cooper pair amplitude $\sim\langle\psi(\epsilon) \psi(\epsilon)\rangle$ must be compensated for by a diminished 'normal state' amplitude $\left\langle\psi^{\dagger}(\epsilon) \psi(\epsilon)\right\rangle$ or, in other words, by a diminished local single particle density of states (see below for a more precise phrasing of this argument.) It is also clear that this suppression mechanism can only be effective for small energies $\epsilon$, and for spatial regions not too distant from the superconductor: For increasing $\epsilon$ or, equivalently, increasing length of the characteristic paths $\gamma$, the action difference $S_{\gamma}(\epsilon)-S_{\gamma}(-\epsilon)$ increases, electron and hole run out of phase and the induced pair amplitude randomizes to zero. 


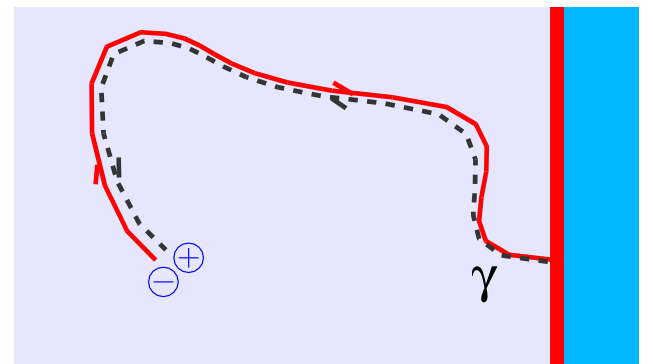

FIG. 1. On the origin of the DoS suppression. The coupling to a superconductor (dark shaded region) leads to the formation of a stable pair amplitude $\sim\langle\psi \psi\rangle$ in the normal metal (light shaded region).

This mechanism of 'action cancellation' is the first of two basic physical principles determining the phenomenology of the proximity effect. It not only explains the formation of a pair amplitude but also the well known sensitivity of the DoS suppression to the classical integrability properties of the system. This latter point is most easily understood within the Bohr-Sommerfeld approach to the problem. The BS approach is based on the condition that the classical action of any closed trajectory traced out by the constituent particles of the system be an integer multiple of $h$. The generic topology of closed trajectories of an SN-system is displayed in Fig. 2: for sufficiently small energies, $\epsilon$, an electron/hole trajectory encompassing two Andreev events closes into itself. The total action of such a trajectory is given by

$$
S_{\gamma}(\epsilon)-S_{\gamma}(-\epsilon)-\frac{h}{2}
$$

where the term $-h$ is due to the Andreev scattering phase shift $i \exp (i \phi)$ 28. Expanding $S_{\gamma}(\epsilon)=S_{\gamma}(0)+T_{\gamma} \epsilon$, where $T_{\gamma}$ is the time of traversal of $\gamma$, one obtains the condition

$$
\frac{2 T_{\gamma} \epsilon}{h} \stackrel{!}{=} n+\frac{1}{2}
$$

where $n$ is an integer. This condition implies that for small $\epsilon$ the structure of the DoS is determined by the distribution $P\left(L_{\gamma}\right)$ of classical paths of large length $L_{\gamma}=$ $v_{F} T_{\gamma}$. (See Refs. 12, 13, 17, 18, for the precise formulation of the BS evaluated DoS.) For classically integrable and classically chaotic systems, respectively, these distributions are qualitatively different. More specifically, for open chaotic systems, $P\left(L_{\gamma}\right) \propto \exp \left(-L_{\gamma} /\left(v_{F} t_{\mathrm{f}}\right)\right)$ is exponentially small (where $t_{\mathrm{f}}$ is the escape time, see section III) whereas for open integrable systems $P\left(L_{\gamma}\right)$ decays only algebraically. (Here the attribute 'open' means that we are dealing with systems that are connected to outside leads.) The exponential rareness of long trajectories provides the BS explanation [18] for the smallness of the low energy DoS in chaotic systems.

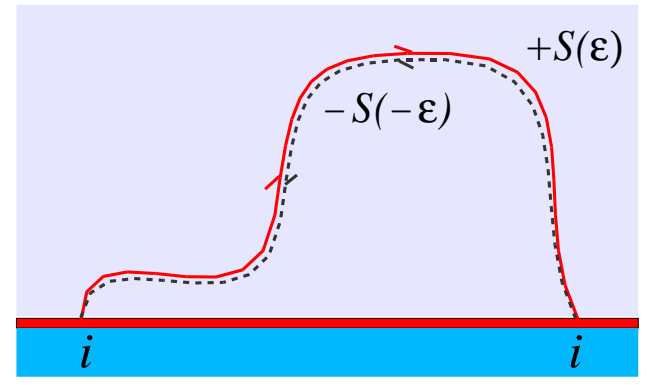

FIG. 2. Generic structure of the classical trajectories forming the basis of the BS scheme.

Notice, however, that irrespective of the structure of the system, there will always be some finite probability $P(L)$ of finding trajectories of length $\sim \hbar v_{F} / \epsilon$. In other words, although the BS prediction for the DoS may be small, it will never be strictly vanishing (for any finite $\epsilon$.) For chaotic systems, this is in contradiction with the results obtained by other methods. Specifically, for diffusive metallic systems [6 10,29, 30], weakly disordered ballistic systems [23], and 'zero-dimensional' quantum dot type systems [12,13] a true DoS gap (strictly vanishing DoS for small energies) has been obtained by quasi-classical, numerical, and random-matrix methods, respectively.

What is the reason for the discrepancy between these results and the BS approach? The answer is that the latter does not account for the second key mechanism relevant for the physics of the proximity effect, quantum diffraction: the discussion above was based on a representation of transition amplitudes in terms of idealized classical trajectories. However, in a quantum system the uncertainty principle imposes an upper cutoff $\hbar$ on the phase space resolution. In practical terms this means that the propagation along the trajectories entering the BS counting scheme must be interpreted as a caricature of the propagation of quantum minimal wave packets. The transverse spreading of these packets is at least of the order of the Fermi wave length $\lambda_{F}$ (cf. Fig. [3).

Whereas in integrable systems this quantum spreading is of no real concern, its consequences in chaotic systems are profound. The existence of a globally positive Liapunov exponent $\lambda$ entails that a microscopic initial uncertainty of $O\left(\lambda_{F}\right)$ increases exponentially $\sim \lambda_{F} \exp (t \lambda)$ and, after a time $t_{\mathrm{E}}=\lambda^{-1} \ln \left(a / \lambda_{F}\right)$, reaches the scale $a$ of the macroscopic geometry of the system. On time scales beyond this so-called Ehrenfest time, a classification scheme based on classical trajectories becomes meaningless. The consequences of this spreading of minimal wave packages have been explored in AL. In that paper a mechanism was discussed by which the spreading leads to the formation of weak localization type quantum corrections to the classical (Drude) conductance of chaotic systems: in the terminology of semiclassics, two initially close trajectories split up but eventually recombine to give an overall phase-insensitive weak localization 
correction.

A similar mechanism affects the mean DoS of SN systems. However, unlike the weak localization corrections to the normal conductance - generally a small correction to a large classical background - quantum diffraction in chaotic SN systems is of vital relevance for even the leading order, qualitative structure of the DoS. In particular, it is responsible for the strict vanishing of $\nu(\epsilon)$ below a certain threshold energy.

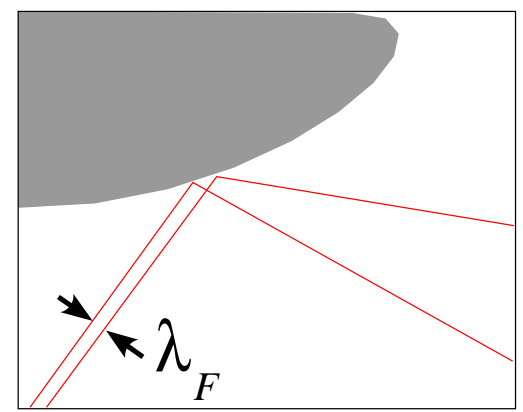

FIG. 3. Quantum diffraction in a chaotic system: after a time set by the largest Liapunov exponent of the system, two initially close trajectories diverge.

For a particular system class, such as diffuse disordered systems, the effect of these quantum contributions can be investigated in quantitative detail. The idea is to compare the trajectory oriented picture outlined above with quantitative solutions obtained by other methods. Indeed, the mean DoS of of a large variety of SN systems with diffusive $\mathrm{N}$ compounds has been obtained by solving the quasiclassical Usadel equations 22,6-10,32. Mathematically, the Usadel equations are second order nonlinear differential equations for the average Green function of the system. They are obtained by a quasiclassical expansion of the microscopic Gorkov equations, with self consistent account for the (quantum) scattering off impurities. Solving these equations it has been found that for systems with an $\mathrm{N}$ region of finite extension $L$, the DoS profile universally contains a hard gap of the type schematically drawn in Fig. 4. We next address the question of how this phenomenon can be reconciled with the semiclassical picture discussed above.

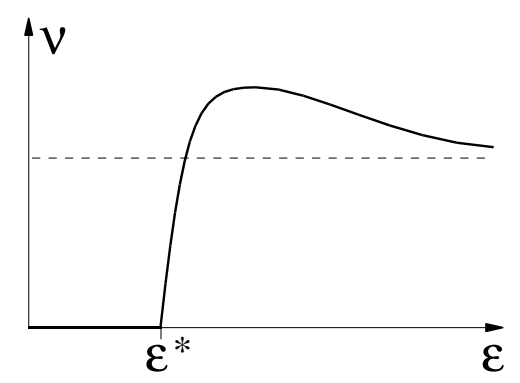

FIG. 4. Qualitative structure of the mean DoS of a compact diffusive system in contact with a superconductor.
For concreteness, let us consider a diffusive system in the quantum dot regime, i.e. a regime where the Thouless energy $E_{c}=\hbar D / L^{2}$ (where $D$ is the diffusion constant) is the largest energy scale in the problem. (The discussion can be adapted straightforwardly to other types of system configurations.) In this case, the Usadel equation is controlled by the dimensionless parameter $\kappa \equiv \gamma / \epsilon$, where $\gamma$ is an energy scale representing the coupling strength to the superconductor. Although the parameter $\kappa$ need not be small, it is nevertheless instructive to expand the Usadel equation in $\kappa$; not to obtain quantitative results, but rather to understand the basic physical mechanism responsible for the buildup of the gapped DoS. Referring to Ref. [10] for a more detailed discussion we here merely sketch the qualitative picture.

The linearity of $\kappa$ in both the coupling to the superconductor and in $1 / \epsilon$ means that each power of this parameter corresponds to a single Andreev scattering event, coupled to a diffusion mode [33]. The diffusion mode describes the coherent propagation of a particle and a hole through a diffusive background (Fig. 5, right inset), i.e. it is the diffusive implementation of the interfering particle/hole amplitudes discussed above. A cartoon of the correction to the DoS of the lowest order in $\kappa$ is depicted in Fig. 5, right. Technically, this correction is described by a single diffusive ladder diagram with two Andreev scattering vertices [10]. However, as our present discussion serves a purely qualitative purpose, we are not going to elaborate on the diagrammatic interpretation any further. The only thing to be kept in mind is that a perturbative semiclassical/diagrammatic approach to calculating the DoS amounts to an expansion in powers of the parameter $\kappa$.

Already at the next to leading order in $\kappa$ contributions of a different topology appear (Fig. 5, center). The distinguishing feature of these corrections is that they contain 'junction' points at which a pair of interfering particle/hole amplitudes splits up (but later recombines to form an overall phase coherent correction). These corrections

- are not included in a semiclassical approach based on a classification in terms of purely classical trajectories, and

- are structurally similar to the weak localization corrections of normal metals. In fact, the basic junction region (left inset in Fig. 5) is a real space representation of what in the context of disordered systems is called a Hikami box. Within diagrammatic approaches these objects appear as vertices in the diagrams describing metallic weak localization.

- Finally, the diffractive splitting of a coherent particle/hole amplitude is a process that does not hinge on the diffusivity of the medium, but merely on the chaoticity of the underlying dynamics. This point will be discussed in great detail in section $\mathrm{VI}$ below. 
The main difference to standard weak localization is that the DoS 'corrections' depicted in Fig. 5 are not small. In fact each additional junction introduces two more powers of $\kappa$, and this parameter can assume arbitrary values. As $\epsilon$ is lowered and $\kappa \nearrow 1$, a situation arises where diffraction processes of infinite order have to be taken into account, a task which can clearly cannot be accomplished within a semiclassical trajectory oriented approach. In fact it turns out that for $\kappa \geq 1$ expansions in terms of trajectories (diagrams) do not even converge in principle.

To understand the origin of the problem, it is instructive to compare the semiclassical expansion with the explicit solution of the Usadel equation. For the quantum dot type system presently under consideration, the quasiclassical expression of the DoS 12 16 is given by the simple expression,

$$
\nu(\kappa)=\nu_{0} \operatorname{Re} \frac{1}{\sqrt{1-\kappa^{2}}},
$$

where $\nu_{0}$ is the bare metallic density of states (in the absence of a superconductor). For our purposes, the most important feature of this expression is its BCS type essential singularity at $\kappa=1$. The structure of this singularity implies that (a) $\kappa=1$ marks the DoS gap, for (b) values $\kappa>1$ the DoS strictly vanishes and (c) the gap region $\kappa>1$ is out of reach of a semiclassical/diagrammatic perturbative expansion centered around the metallic limit $\kappa \rightarrow 0$. For other types of diffusive systems, the relevant control parameter may be a different one. Also the analytical structure of the quasiclassical DoS will in general be way more complicated than the expression above [6 10]. However the three points (a)-(c) remain generally valid.

In the rest of the paper we will try to explore to what extent these structures generalize from diffusive to more general system classes. This analysis will be formulated within a hybrid formalism comprising quasiclassical Green function methods and elements of the supersymmetry approach to disordered systems. The relevant theoretical background material is reviewed in the next two sections.

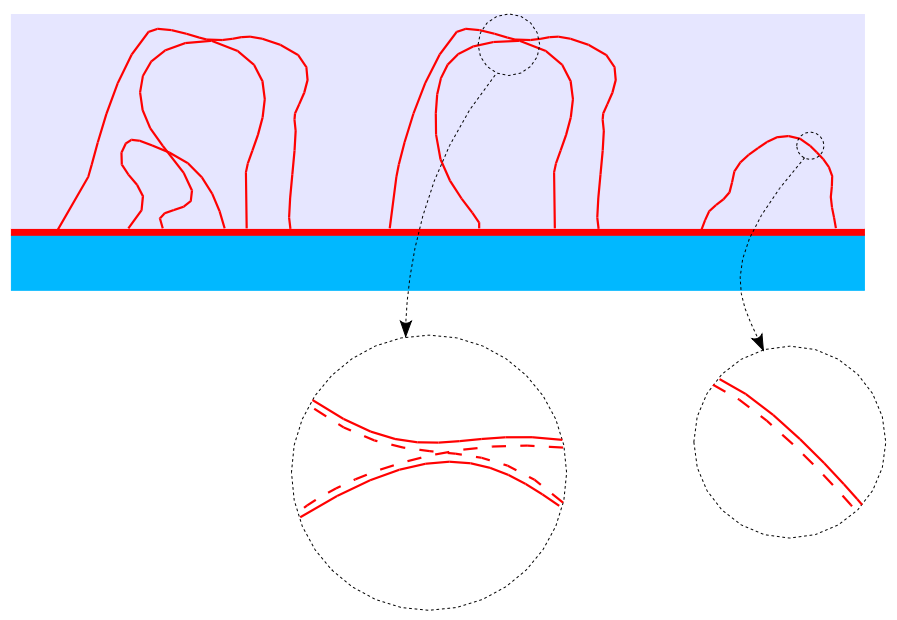

FIG. 5. On the semiclassical interpretation of the solution of the Usadel equations.

\section{QUASICLASSICAL FORMULATION}

To promote the discussion of the previous section to a more quantitative level, we start out from the Eilenberger equation [34]. While the Eilenberger equation may be written in a general form that describes either point-like or long-ranged scatterers, we focus in this section on its application to systems with hard boundaries and weakly disordered by point-like scatterers ('disordered billiards'). The case of clean systems with soft potentials will be discussed more fully in section VI.

The general form of the Eilenberger equation may be written as

$$
\begin{gathered}
\left(\hbar \mathcal{L}-i\left[\sigma_{3}(\epsilon+\hat{\Delta}(\mathbf{r})),\right]\right) g(\mathbf{r}, \mathbf{n})=\mathcal{O}_{\text {coll }}[g] \\
g(\mathbf{r}, \mathbf{n}) g(\mathbf{r}, \mathbf{n})=\mathbb{1},
\end{gathered}
$$

where $g(\mathbf{r}, \mathbf{n})$ is the quasiclassical retarded Green function of the system 34, 35, $\mathcal{L}$ the classical Liouville operator, $\mathcal{L}=\{H, \quad\}$ acting in the phase space of points $(\mathbf{r}, \mathbf{p})$, and $H$ the Hamiltonian function of the system. The coordinates $\mathbf{x} \equiv(\mathbf{r}, \mathbf{n})$ parameterize a $2 d-1$ dimensional shell of constant energy $E_{F}=p_{F}^{2} /(2 m)$, where $\mathbf{n}=\mathbf{p} / p_{F}, p_{F}$ is the Fermi momentum and $d$ the dimensionality of the system. Further, $\sigma_{i}$ are Pauli matrices operating in the two component particle-hole ( $\mathrm{PH})$-space, and $\hat{\Delta} \equiv \Delta\left(\cos \phi \sigma_{1}+\sin \phi \sigma_{2}\right)$ is the Nambu representation of the phase dependent order parameter. For an SN system the support of $\hat{\Delta}$ is limited to the $\mathrm{S}$ region where it must, in principle, be determined self consistently. With regard to the the structure of the proximity induced gap in the $\mathrm{N}$ region, however, the details of the order parameter profile in $\mathrm{S}$ are of secondary importance, and it suffices to model $\Delta$ by a step function

$$
\Delta(\mathbf{r})=\left\{\begin{array}{ll}
\Delta & \mathbf{r} \in \mathrm{S}, \\
0 & \mathbf{r} \in \mathrm{N}
\end{array} .\right.
$$

For simplicity we will assume that the superconductor has a constant order parameter phase $\phi=0$.

The operator $\mathcal{O}_{\text {coll }}[g]$ appearing on the right-hand side of equation (11) describes the effect of impurity scattering. Its functional form depends on the microscopic realization of the scattering potential:

$$
\begin{aligned}
\mathcal{O}_{\text {coll }}[g] & =-\frac{\hbar}{2} \int \frac{d \mathbf{n}^{\prime}}{S_{d}}\left[w\left(\mathbf{n}-\mathbf{n}^{\prime}\right) g\left(\mathbf{r}, \mathbf{n}^{\prime}\right), g(\mathbf{r}, \mathbf{n})\right] \\
w\left(\mathbf{n}-\mathbf{n}^{\prime}\right) & =2 \pi \nu n_{i}\left|\tilde{V}\left(\mathbf{n}-\mathbf{n}^{\prime}\right)\right|^{2}
\end{aligned}
$$

where $S_{d}$ is the surface of the $d$-dimensional unit sphere. Here $\tilde{V}$ is the Fourier transform of the scattering amplitude and $n_{i}$ is the impurity concentration, while as usual the limits of $\tilde{V} \rightarrow 0$ and $n_{i} \rightarrow \infty$ are implied, such that 
$n_{i}|V|^{2}$ is constant. The scattering lifetime $\tau$ is defined through $w\left(\mathbf{n}-\mathbf{n}^{\prime}\right)$ by

$$
\frac{1}{\tau}=\int \frac{d \mathbf{n}^{\prime}}{2 \pi} w\left(\mathbf{n}-\mathbf{n}^{\prime}\right) .
$$

For the weakly disordered systems considered in this paper, the scattering time exceeds the typical time of flight through the system.

For finite-size impurities characterized by some Gaussian potential envelope function, we have

$$
w\left(\mathbf{n}-\mathbf{n}^{\prime}\right) \propto \exp \left(-\frac{p_{F}^{2} a^{2}}{\hbar^{2}}\left|\mathbf{n}-\mathbf{n}^{\prime}\right|^{2}\right),
$$

where $a$ is the typical extent of the scattering region. In the remainder of this section we will focus on an ultra-quantum limit obtained by sending $\hbar$ to infinity at fixed Fermi energy $E_{F}$ and fixed $a$. (Notice the differences with the limit of $\hbar \rightarrow 0$ at fixed electron density $\propto\left(p_{F} / \hbar\right)^{d}$, usually taken in Boltzmann transport theories.) In this limit the form factor $w$ becomes independent of the momentum transfer $\propto \mathbf{n}-\mathbf{n}^{\prime}$ : hence this limit corresponds to purely $s$-wave scattering. The motivation for considering the $s$-wave limit first is that the impurity scattering becomes maximally diffractive, making it an ideal test case for studying the impact of quantum effects on the DoS-profile. Indeed, for $s$-wave impurities, it is the ordinary scattering time $\tau$ that sets the time scale after which classical trajectories are destabilized through diffraction and one expects $\hbar / \tau$ to be relevant energy scale for the DoS gap. More complex mechanisms of diffraction will be considered in section $\mathrm{VI}$ when we discuss the proximity effect in chaotic systems.

As a preparation to the discussion of the full Eilenberger equation (1) it is instructive to consider the diffractionless limit, for which $\tau \rightarrow \infty$. Physically, this limit describes the regime of large energies, $\epsilon \gg \hbar / \tau$ (time scales much shorter than the scattering time) for which the collision operator is negligibly weak as compared to both the order parameter and the energy argument. In the limit of no diffraction, the Eilenberger equation reduces to an ordinary first order differential equation that can be solved analytically [17]. Indeed, each phase space point $\mathbf{x}$ can unambiguously be assigned to a classical trajectory $\mathbf{x}(t)$ passing through it. The initial and final point of this trajectory are set by two points of Andreev reflection (cf. Fig. 2). Denoting the time of the first (second) Andreev scattering event by $-(+) t_{0} / 2$ and using that $\dot{\mathbf{x}}=\{H, \mathbf{x}\}$, the Eilenberger equation in the normal region reduces to

$$
\left(\hbar d_{t}-i \epsilon\left[\sigma_{3},\right]\right) g(\mathbf{x}(t))=0, \quad t \in\left[-t_{0} / 2, t_{0} / 2\right] .
$$

In the limit $\Delta / \epsilon \rightarrow \infty$ the solution of this equation is given by [17],

$$
g(\mathbf{x}(t))=\sin \theta \cos \left(\frac{\epsilon t}{\hbar}\right) \sigma_{2}+\cos \theta \sigma_{3}-\sin \theta \sin \left(\frac{\epsilon t}{\hbar}\right) \sigma_{2},
$$

where $\cos \theta=-i \tan \left(\epsilon t_{0} / \hbar\right)$. This expression contains two pieces of information that will be of key importance for all what follows: (i) The non-diffractive Eilenberger formalism is equivalent to the BS-approach 12, 13, 17, 18]. To derive this equivalence one starts out from the general formula for the DoS,

$$
\nu(\epsilon)=\frac{\nu_{0}}{2} \operatorname{Re} \int d \mathbf{x} \operatorname{tr}\left(g\left(\mathbf{x}, \epsilon^{+}\right) \sigma_{3}\right),
$$

where $\nu_{0}$ is the metallic reference DoS and the phase space integral $\int d \mathbf{x}=1$ is normalized to unity. Substituting the clean solution for $\epsilon^{+}=\epsilon+i 0$ it is a straightforward matter to show that

$$
\nu(\epsilon) \stackrel{\tau \rightarrow \infty}{=} \pi \nu_{0} \int d \mathbf{x} \sum_{n=-\infty}^{\infty} \delta\left(\frac{\epsilon t_{0}(\mathbf{x})}{\hbar}-\left(n+\frac{1}{2}\right) \pi\right)
$$

which is the BS result 12, 13, 17, 18. (ii) For energies $\epsilon \ll \hbar / t_{0}(\mathbf{x})$ much smaller than the inverse flight time through the normal segment of the trajectory, $g(\mathbf{x}) \approx \sigma_{2}$ is approximately 'locked' to the order parameter of the adjacent superconductor. This fact will become of importance below when we analyze the full Eilenberger equation.

We now proceed to the discussion of diffraction effects. For finite $\tau$, the Eilenberger equations along different trajectories become coupled and no rigorous solution is possible. In chaotic systems, however, one may invoke statistical arguments to turn the complexity of the problem to an advantage. (Notice that under the conditions specified above, the presence of impurities alone suffices to render the dynamics chaotic on time scales $t>\tau$.) More specifically, we are going to exploit the features of the path length distribution function $P\left(v_{F} t_{0}\right)$ of systems with chaotic dynamics to propose a trial solution of the Eilenberger equation that should be close to the unknown exact solution. Contrary to the BS result, the DoS derived from the approximate solution will exhibit a hard gap. In the next section we will then show that this gap is robust to all orders of perturbation theory around the trial solution.

We begin by noticing that in the diffractive problem two intrinsic time scales exist: The scattering time $\tau$ and the 'typical escape time' $t_{\mathrm{f}}$, i.e. the time scale $t_{\mathrm{f}}=L_{\mathrm{f}} / v_{F}$ corresponding to the maximum $L_{\mathrm{f}}$ of the path length distribution function $P(L)$. For a ballistic system, we have $\tau \gg t_{\mathrm{f}}$, and so we conclude that three qualitatively different temporal regimes exist: for short times $t \ll t_{\mathrm{f}}$, both diffraction effects and the differences between integrable and chaotic dynamics are negligible, and the DoS is well approximated by the BS formula. For intermediate times $t_{\mathrm{f}} \ll t \ll \tau$ diffraction is still inessential but the structure of the function $P(L)$ begin to matter. Specifically, the exponential rarity of trajectories with $t_{0}>t_{\mathrm{f}}$ implies a strongly suppressed DoS. Finally, for times $t \gg \tau$, diffraction is the dominant physical process. 
Technically, the relevancy of diffraction follows from the fact that for small energies $\epsilon \ll \hbar / \tau$ the scattering operator is the dominant term in the Eilenberger equation of the normal region. For any given real space point, $\mathbf{r}$, the scattering term involves an average over all trajectories passing through $\mathbf{r}$. Apart from some exponentially rare exceptions, the vast majority of these trajectories will have a flight time of the order $t \approx t_{\mathrm{f}} \ll \hbar / \epsilon$. For all these trajectories, the solution of the clean Eilenberger equation is approximately given by $\sigma_{2}$, i.e. set by the bulk value of the order parameter. This justifies the approximation of the collision operator by $\mathcal{O}_{\text {coll }} \approx$ $-\hbar(2 \tau)^{-1}\left[\sigma_{2},\right]$. Physically, $\mathcal{O}_{\text {coll }}$ then acts like an induced order parameter of strength $\approx \hbar /(2 \tau)$. This effective order parameter field in the $\mathrm{N}$ region describes the large angle scattering of Andreev states off the induced proximity pairing field amplitude. (Notice the difference between a pairing field amplitude, $\langle\psi \psi\rangle$, and an order parameter, which is given by the coupling constant $\times\langle\psi \psi\rangle$ ).

The effect of $\mathcal{O}_{\text {coll }}$ on the solution of the equation depends on the length of the trajectory under consideration. For trajectories with $t_{0} \ll \tau$, the clean solution (2) is close to the bulk form set by the presence of the superconductor at the boundaries anyway, and the extra presence of the diffraction operator is of no qualitative significance. For the sparse set of exceptionally long trajectories with $t_{0}>2 \tau$, however, the boundary condition is largely inessential and the equation is governed by a competition between the energy and the collision operator in the bulk $\mathrm{N}$ region. Inspection of the equation shows that the solution will approximately be of standard BCS type

$$
\left.g(\mathbf{x})\right|_{t_{0}(\mathbf{x})>\tau} \stackrel{\epsilon<\hbar /(2 \tau)}{\approx} \frac{1}{\sqrt{(\hbar / 2 \tau)^{2}-\epsilon^{2}}}\left(i \epsilon \sigma_{3}+\frac{\hbar}{2 \tau} \sigma_{2}\right) .
$$

Eq. (3) leads to vanishing DoS for energies $\epsilon<\hbar /(2 \tau)$.

Summarizing we have formulated a primitive trial solution $g_{0}(\mathbf{x}, \epsilon)$ to the Eilenberger equation that predicts a gapped DoS. For trajectories $t_{0}(\mathbf{x}) \ll \tau$ (energies $\epsilon \gg \hbar / \tau), g_{0}$ coincides with the diffractionless solution (2). Only for very long trajectories the non-diffractive asymptotics is cut off by the collision operator, leading to the trial solution of Eq. (3). We emphasize that the simple BCS solution (3) is specific to the case of purely $s$ wave scattering and becomes unreliable in the vicinity of the gap, $\epsilon \approx \hbar /(2 \tau)$. In the next section we will explore the sensitivity of the result to perturbative corrections around the trial solution. It will turn out that the vanishing of the DoS below the gap is a phenomenon robust to infinite order in perturbation theory.

\section{FIELD THEORETIC FORMULATION}

The previous discussion raises the question of whether the DoS gap represents a generic feature of disordered billiards systems or whether it is an artefact of the BCS type low energy trial solution derived above. In principle, one may address this issue by using the trial solution as a starting point for an infinite order perturbative expansion of the Eilenberger equation. (Given that we are looking for residual corrections to a vanishing DoS, any conclusive perturbative approach has to be driven to infinite order.) In practice, however, such a type of perturbative expansion is neither easy to formulate nor does it provide much physical insight. Given this situation we find it more convenient to follow a different approach. This alternative route, which will be based on a variational principle, has three advantages: (i) the variational approach will provide us with a comparatively transparent verification of the robustness of the gap, (ii) it draws upon analogies between the current problem and more general recent developments 25,26,36] regarding the dynamics of chaotic mesoscopic systems, (iii) it will provide the theoretical background for the subsequent analysis of clean chaotic SN systems.

What is the meaning of the phrase "variational principle' in the present context? Within the framework of normal systems, Khmelnitskii and Muzykhantskii have found [25] a general scheme whereby quasiclassical kinetic equations are obtained through a variational analysis of certain functional integrals of nonlinear $\sigma$-model type. Below we will introduce the (straightforward) extension of this scheme to the case of superconductor problems. Thereafter the functional integral formalism will be applied to an analysis of the DoS. By integrating out fluctuations around the trial solution discussed in the previous section, we will find that the gap survives.

To discuss the variational principle, we need to introduce some field theory oriented formalism which, at first sight, might appear to be quite unrelated to the previously discussed material: Consider the functional integral

$$
Z[\hat{J}] \equiv \int \mathcal{D} T e^{-S[T]},
$$

where the action $S[T]=S_{\mathrm{reg}}[T]+S_{\mathrm{coll}}[T]$ with

$$
\begin{aligned}
& S_{0}[T]=-\pi \rho \int d \mathbf{x} \operatorname{str}\left(\hbar T \sigma_{3} \mathcal{L} T^{-1}+\right. \\
& \left.\quad+i \sigma_{3}(\epsilon+\hat{\Delta}) Q+\hat{J} \otimes E_{11}^{\mathrm{BF}} Q\right), \\
& S_{\mathrm{coll}}[T]=\frac{\pi \hbar \rho}{4} \int \frac{d \mathbf{r} d \mathbf{n} d \mathbf{n}^{\prime}}{L^{d}\left(S_{d}\right)^{2}} w\left(\mathbf{n}, \mathbf{n}^{\prime}\right) \operatorname{str}\left(Q(\mathbf{r}, \mathbf{n}) Q\left(\mathbf{r}, \mathbf{n}^{\prime}\right)\right) .
\end{aligned}
$$

Here $T(\mathbf{x})$ and $Q(\mathbf{x})=T(\mathbf{x}) \sigma_{3} T(\mathbf{x})^{-1}$ are four dimensional supermatrix fields in phase space. As before, the (unit-normalized) integration extends over a phase space shell of constant energy $E_{F}$, and the inverse level spacing at $E_{F}$ is denoted by $\rho$. The rest of the notation is adopted to conventions standard in the literature of the nonlinear $\sigma$-model [37]: The field $T$ takes values in 
the (maximally Riemannian subset) of the graded coset space $\mathrm{GL}(2 \mid 2) /(\mathrm{GL}(1 \mid 1) \otimes \mathrm{GL}(1 \mid 1))$. In an explicit index representation, $T(\mathbf{x})=\left\{T_{a \alpha, b \beta}(\mathbf{x})\right\}$ where the indices $\alpha, \beta=1,2$ discriminate between the bosonic and fermionic blocks of $T$ and the index $a, b=1,2$ is the $\mathrm{PH}$ index. 'str' is the standard supertrace defined through $\operatorname{str}(A)=-\sum_{a, \alpha} A_{a \alpha, a \alpha}(-)^{\alpha}$ (which differs by an overall minus sign from Efetov's convention.) Finally, the two dimensional matrix field $\hat{J}=\left\{J_{a b}(\mathbf{x})\right\}$ serves as a source field to generate correlation functions, and $E_{11}^{\mathrm{BF}}$ is defined as $\left(E_{11}^{\mathrm{BF}}\right)_{i j}=\delta_{i 1} \delta_{j 1}$.

An action of the structure of (雨) has first been introduced in the context of normal systems with weak impurity scattering [25]. (For an alternative derivation, see Ref. [26].) For a normal system, $\hat{\Delta}=0$ and the action describes the correlated propagation of an retarded and an advanced Green function in a ballistically disordered environment. (The role of the indices $a, b$ then is to discriminate between the advanced and the retarded sector of the theory.) Technically, the functional integral $Z[\hat{J}]$ is of nonlinear $\sigma$-model type, so that Eq. (4) is commonly designated as the action of the 'ballistic $\sigma$-model'.

In the context of superconductivity, the model describes a single impurity averaged Gorkov Green function. More specifically,

$$
\begin{aligned}
G(\mathbf{r}, \mathbf{n}) & =\left.\frac{1}{\pi \nu_{0}} \frac{\delta Z[\hat{J}]}{\delta \hat{J}(\mathbf{r}, \mathbf{n})}\right|_{\hat{J}=0} \\
& =\left\langle Q_{11}(\mathbf{r}, \mathbf{n})\right\rangle_{Q},
\end{aligned}
$$

where $\nu_{0}=\rho / L^{d}$ and $G(\mathbf{r}, \mathbf{n})$ is the energy-integrated Wigner transform of the microscopic Gorkov Green function [35]. Further, the indices in $Q_{11}$ refer to BF space, and $\langle\ldots\rangle_{Q}$ denotes functional averaging with respect to the source free action $S[Q]$. From (5) the DoS is obtained as

$$
\nu=\frac{\nu_{0}}{2} \int d \mathbf{r} d \mathbf{n} \operatorname{Re}\left\langle\operatorname{tr}\left(Q_{11}(\mathbf{r}, \mathbf{n}) \sigma_{3}\right)\right\rangle_{Q} .
$$

The derivation of both the effective action (住) and the key relation (5) is discussed in Appendix A. We here merely notice that the construction of the model involves essential elements of the quasiclassical approach. In fact, the derivation essentially amounts to an implementation of the quasiclassical construction steps - disorder average, Wigner transform, and integration over the modulus of the momentum $p$ - into a functional integral approach. (The additional graded structure of the functional integral is physically of marginal relevance; supersymmetry merely ensures unit normalization $Z[\hat{J}=0]$ of the source free partition function. In fact, this aspect will not be of much importance in our further analysis.)

What is the connection between the field integral approach and the Eilenberger equation and, closely related, why contemplate a functional integral in a situation that is well described by quasiclassics? To address these issues, we first notice that the functional integral $\hat{Z}[\hat{J}]$ cannot be evaluated in closed form. It is, however, a good candidate for a stationary phase approach. (This is because in the present application the coupling constants appearing in the action are much larger than unity.) As usual with nonlinear $\sigma$-models, the action $S[T]$ possesses not just one but rather an entire manifold of degenerate saddle points (a fact that readily follows from the observation that the action is invariant under transformations in boson/fermion (BF) space.) We start out by seeking for those saddle points $\bar{Q}$ whose matrix structure is as simple as possible. Given the BF invariant structure of the action, one anticipates that these saddle points will be proportional to unity in BF space, $\bar{T}=\left\{\bar{T}_{a b} \delta_{\alpha \beta}\right\}$.

Defining $\bar{Q} \equiv \bar{T} \sigma_{3} \bar{T}^{-1}$, it is a straightforward matter to show that the variation of the source free action leads to

$$
\begin{aligned}
& \frac{\delta S[\bar{T}]}{\delta \bar{T}(\mathbf{x})}=0 \Rightarrow \\
& \quad \Rightarrow \hbar \mathcal{L} \bar{Q}(\mathbf{x})-i\left[\sigma_{3}(\epsilon+\hat{\Delta}(\mathbf{x})), \bar{Q}(\mathbf{x})\right]=\mathcal{O}_{\text {coll. }}[\bar{Q}]
\end{aligned}
$$

where

$$
\mathcal{O}_{\text {coll. }}[\bar{Q}]=-\frac{\hbar}{2} \int \frac{d \mathbf{n}^{\prime}}{S_{d}} w\left(\mathbf{n}, \mathbf{n}^{\prime}\right)\left[\bar{Q}\left(\mathbf{r}, \mathbf{n}^{\prime}\right), \bar{Q}(\mathbf{r}, \mathbf{n})\right] .
$$

The above equations state that the (BF diagonal) mean field configuration of the functional integral are determined by the Eilenberger equation or, equivalently, that $\bar{Q}=g$ is to be identified with the quasiclassical Green function. This connection can be made explicit by evaluating (5) on the diagonal saddle point level which gives

$$
G(\mathbf{r}, \mathbf{n}) \simeq e^{-S[\bar{Q}]} \bar{Q}(\mathbf{r}, \mathbf{n})=\bar{Q}(\mathbf{r}, \mathbf{n})=g(\mathbf{r}, \mathbf{n}),
$$

where the second equality is based on the fact that (due to supersymmetry) the action $S[\bar{Q}]=0$.

Another way of interpreting this result is to say that the ballistic $\sigma$-model implements a variational principle of quasiclassics, a fact first realized in Ref. [25] (with reference to normal systems.) In the context of superconductivity this observation is of significant practical relevance, the reason being that the variational action (4) turns out to be a tool more flexibly applicable than the Eilenberger equation itself. More specifically,

- the field integral approach enables one to include quantum fluctuations around the mean field extremum. As was shown in Ref. [10] (for the case of the dirty limit, $\Delta<\hbar / \tau)$ such fluctuations may lead to a renormalization of the leading order quasiclassical results.

- it is straightforward to extend the field integral formulation to the computation of the disorder average of more than one Green function. This enables one to analyze mesoscopic fluctuation effects [10]. 
- in cases where the exact solution of the Eilenberger equation is unknown one may still organize the functional integral around an approximate mean field solution. Provided that this configuration is not separated from the true Eilenberger solution by points of non-analyticity, no information is lost and a (sufficiently accurate) integration over fluctuations around the trial solution will produce correct results.

For our present application, the third of the above points is of relevance. To explore the DoS at low energies $\epsilon$ one may perform a functional integration around an approximate solution of the Eilenberger equation. In the next section this scheme will be applied to the disordered billiards discussed in section III. In section VI we will then extend the discussion to systems with smooth potentials.

\section{SPECTRUM OF AN SN-SYSTEM WITH BALLISTIC DISORDER}

In this section we apply the strategy outlined at the end of the previous section to explore the DoS of a disordered billiard (with $s$-wave impurities) for energies $\epsilon \ll \hbar / \tau$. For these low energies, it is convenient to organize the functional integration around the reference configuration

$$
Q_{\mathrm{r}} \equiv R \sigma_{3} R^{-1}=\sigma_{2},
$$

where the rotation matrix is

$$
R \equiv \exp \left(i \frac{\pi}{4} \sigma_{1}\right) .
$$

Comparison with the discussion of section III (Eqs. (2) and (3)) indeed shows that for $\epsilon \ll \hbar / \tau$, this configuration is close to the exact Eilenberger solution (see below on what precisely is meant by 'close' in the present context.) Alternatively, one might organize the integration around the trial solution (3). For practical reasons, however, $Q_{\mathrm{r}}=\sigma_{2}$ is a more convenient starting point.

To include fluctuations around $Q_{\mathrm{r}}$, we next introduce the parameterization

$$
Q \equiv R \tilde{Q} R^{-1}, \quad \tilde{Q} \equiv T \sigma_{3} T^{-1},
$$

where $T(\mathbf{x})$ is a four-dimensional supermatrix field on the constant energy shell in phase space. Substitution of this ansatz into the action of the functional integral leads to

$$
\begin{aligned}
& S_{0}[T]=-\pi \rho \int_{\mathrm{N}} \frac{d \mathbf{r}}{V} \int \frac{d \mathbf{n}}{S_{d}} \operatorname{str}\left(\hbar v_{F} \mathbf{n} \cdot T \sigma_{3} \partial_{\mathbf{r}} T^{-1}-\right. \\
& \left.\quad-i \epsilon \sigma_{2} \tilde{Q}\right), \\
& S_{\text {coll }}[T]=\frac{\pi \hbar \rho}{4 \tau} \int_{\mathrm{N}} \frac{d \mathbf{r}}{V} \int \frac{d \mathbf{n} d \mathbf{n}^{\prime}}{S_{d}^{2}} \operatorname{str}\left(\tilde{Q}(\mathbf{r}, \mathbf{n}) \tilde{Q}\left(\mathbf{r}, \mathbf{n}^{\prime}\right)\right) .
\end{aligned}
$$

The functional expectation value associated with the Green function assumes the form

$$
G(\mathbf{x})=\pi \nu_{0}\left\langle\left(R \tilde{Q} R^{-1}\right)_{11}(\mathbf{x})\right\rangle_{\tilde{Q}},
$$

which leads to

$$
\nu=-\frac{\nu_{0}}{2} \int d \mathbf{x} \operatorname{Re}\left\langle\operatorname{str}\left(\tilde{Q}_{11}(\mathbf{x}) \sigma_{2}\right)\right\rangle_{\tilde{Q}},
$$

for the DoS in the normal region. In (6) we have used that for the problem under consideration the Liouville operator $\mathcal{L}$ assumes the form $v_{F} \mathbf{n} \cdot \partial_{\mathbf{r}}$. The symbol $\int_{\mathrm{N}} d \mathbf{r}$ indicates that the integration is restricted to the N-region of the system (with volume $V$ ). At the boundary to the superconductor, we have $T=\mathbb{1}$, which implies that $Q$ becomes tightly locked to its bulk superconductor value, $\sigma_{2}$. Comparison with the general expression (4) shows that (i) due to transformation by $R$ the Pauli matrix multiplying the energy argument has rotated to the superconductor reference point $\left(\sigma_{3} \rightarrow-\sigma_{2}\right)$, and that (ii) in Eq. (6) the appearance of the order parameter is implicit, namely through the boundary condition imposed at the SN interface.

To prepare the integration over fluctuations, we next introduce the standard [37] system of coordinates $T=$ $1+W / 2$, where the matrices $W$ are defined through

$$
W=\left(\begin{array}{cc} 
& B \\
B &
\end{array}\right)
$$

the block decomposition is in $\mathrm{PH}$ space and the $2 \times 2$ supermatrices $B$ are subject to the (convergence ensuring) relation

$$
\bar{B}=\sigma_{3}^{\mathrm{BF}} B^{\dagger}
$$

We substitute this representation into the action (6) and expand formally according to

$$
S[T] \rightarrow S[B, \bar{B}] \equiv \sum_{n=1}^{\infty} S^{(n)}[B, \bar{B}],
$$

where $S^{(n)}[B, \bar{B}]$ is the contribution of $n$th order in $B, \bar{B}$ to (6). Similarly, the DoS functional expectation value can be represented as

$$
\nu=\frac{\nu_{0}}{2} \int_{\mathrm{N}} d \mathbf{x} \sum_{n=0}^{\infty}\left\langle D^{(n)}[B, \bar{B}]\right\rangle_{B, \bar{B}},
$$

where the operators $D^{(n)}[B, \bar{B}]$ are defined through the series expansion

$$
\operatorname{str}\left(\tilde{Q}_{11}(\mathbf{x}) \sigma_{2}\right)=\sum_{n=0}^{\infty} D^{(2 n+1)}[B, \bar{B}],
$$

and we have noticed that only contributions of odd power in $B$ appear. To prepare the perturbative integration over the $B$ degrees of freedom we next consider the second order operator $S^{(2)}[B, \bar{B}]$ of the action. It is a straightforward matter to verify that the explicit form of $S^{(2)}[B, \bar{B}]$ is given by 


$$
S^{(2)}[B, \bar{B}]=\frac{\hbar \pi \rho}{2} \int_{\mathrm{N}} \frac{d \mathbf{r}}{V} \int \frac{d \mathbf{n}}{2 \pi} \frac{d \mathbf{n}^{\prime}}{S_{d}^{2}} \operatorname{str}\left[B(\mathbf{r}, \mathbf{n})\left(\left(-v_{F} \mathbf{n} \cdot \partial_{\mathbf{r}}+\frac{1}{\tau}\right) \delta\left(\mathbf{n}-\mathbf{n}^{\prime}\right)-\frac{1}{\tau}\right) \bar{B}\left(\mathbf{r}, \mathbf{n}^{\prime}\right)\right] .
$$

The operator

$$
\Pi^{-1} \equiv \frac{\hbar \pi \rho}{2}\left(\left(-v_{F} \mathbf{n} \cdot \partial_{\mathbf{r}}+\frac{1}{\tau}\right) \delta\left(\mathbf{n}-\mathbf{n}^{\prime}\right)-\frac{1}{\tau}\right)
$$

governing the quadratic action has two key structural features (which, as will be discussed in the next section, generalize to different system categories): it is (a) real and (b) possesses a spectrum that is gapped against zero. Whereas the reality of $\Pi^{-1}$ is manifest, the presence of a gap follows from the fact that Dirichlet boundary conditions $B=0$ at the interface to the superconductor are imposed. This implies that the phase space zero mode $B(\mathbf{x})=$ const., the only candidate for a zero energy eigenmode, does not belong to the representation space of $\Pi^{-1}$. It is straightforward to verify that all eigenmodes with a finite variation have eigenvalues of at least $\mathcal{O}(\hbar / \tau)$.

The features (a) and (b) imply that the operator $\Pi^{-1}$ possesses a real and non-singular inverse $\Pi\left(\mathbf{x}, \mathbf{x}^{\prime}\right)$. As we will discuss in a moment, these properties suffice to essentially determine the structure of the low energy DoS.

To perturbatively integrate out the $B$ 's, we employ the supersymmetry implementation [37,36] of Wick's theorem: the basic Gaussian pair contraction of two matrices $B$ and $\bar{B}$ is determined by the rules

$$
\begin{aligned}
& \left\langle\operatorname{str}\left(B(\mathbf{x}) A \bar{B}\left(\mathbf{x}^{\prime}\right) A^{\prime}\right)\right\rangle_{0}=\Pi\left(\mathbf{x}^{\prime}, \mathbf{x}\right) \operatorname{str}(A) \operatorname{str}\left(A^{\prime}\right), \\
& \left\langle\operatorname{str}(B(\mathbf{x}) A) \operatorname{str}\left(\bar{B}\left(\mathbf{x}^{\prime}\right) A^{\prime}\right)\right\rangle_{0}=\Pi\left(\mathbf{x}^{\prime}, \mathbf{x}\right) \operatorname{str}\left(A A^{\prime}\right),
\end{aligned}
$$

where $A$ and $A^{\prime}$ are arbitrary two-dimensional supermatrices, and $\langle\ldots\rangle_{0} \equiv \int \mathcal{D}(B, \bar{B}) \exp \left(-S^{(2)}[B, \bar{B}]\right)(\ldots)$ denotes averaging with respect to the Gaussian action. The Gaussian average

$$
\left\langle\mathcal{O}_{1}^{\left(n_{1}\right)}[B, \bar{B}] \mathcal{O}_{2}^{\left(n_{2}\right)}[B, \bar{B}] \ldots\right\rangle_{0}
$$

of an arbitrary constellation of $n_{k}$ th order operators $\mathcal{O}_{k}^{\left(n_{k}\right)}$ can then, at least in principle, be computed as the sum of all possible total pair contractions.

In general, the infinite series emanating from an expansion of the exponentiated action is far too complex to be perturbatively brought under control. With the current problem, however, the situation is different. It turns out that the series expansion of both the operators entering the action and the preexponential terms are sufficiently highly structured to make possible some categoric (infinite order in perturbation theory) statements. To be more specific, notice that the operators appearing in the action fall into two different categories:

- operators $O_{\mathrm{e}}[T]=\sum_{n} \mathcal{O}_{\mathrm{e}}^{(2 n)}[B, \bar{B}]$ whose expansion contains only even powers of $B$ and which are real (in the sense that the operator content multiplying the $B$ 's is real). The two representatives of this category are the kinetic operator, $\sim \operatorname{str}\left(\mathbf{n} \cdot T \sigma_{3} \partial_{\mathbf{r}} T^{-1}\right)$, and the collision operator $\sim \operatorname{str}\left(\langle\tilde{Q}\rangle_{\mathbf{n}}\langle\tilde{Q}\rangle_{\mathbf{n}}\right)$

- operators of the type $i O_{\mathrm{o}}[T]=\sum_{n} \mathcal{O}_{\mathrm{o}}^{(2 n+1)}[B, \bar{B}]$ where $O_{\mathrm{o}}$ is again real but contains only odd powers of $B$. This class of operators is represented by the energy term, $\sim \operatorname{str}\left(\sigma_{2} \tilde{Q}\right)$.

Similarly we notice that the preexponential expansion (7) belongs to the class of purely odd operators. However, contrary to the operators appearing in the action, the preexponential expansion is purely imaginary (the $i$ 's appearing in the definition of the Pauli matrix $\sigma_{2}$.) Finally, we repeat that the 'propagator' $\Pi$ is real.

With all these things in place we now investigate the perturbative representation of the DoS expectation value. Consider the contribution of any particular operator $D^{(2 n+1)}[B, \bar{B}]$ to the expansion $(\overline{7})$. In order to obtain a non-vanishing contraction, we need to bring down a complementary odd operator from the action. The most general odd operator that can be constructed from the exponentiated action is of the form

$$
i^{k} O_{\mathrm{o}}^{\left(2 n_{1}+1\right)} \ldots O_{\mathrm{o}}^{\left(2 n_{k}+1\right)} O_{\mathrm{e}}^{\left(2 m_{1}\right)} \ldots O_{\mathrm{e}}^{\left(2 m_{l}\right)},
$$

where $l$ is arbitrary but $k$ must be odd. The total contraction

$$
\begin{gathered}
\left\langle D^{(2 n+1)} O_{\mathrm{o}}^{\left(2 n_{1}+1\right)} \ldots O_{\mathrm{o}}^{\left(2 n_{k}+1\right)} O_{\mathrm{e}}^{\left(2 m_{1}\right)} \ldots O_{\mathrm{e}}^{\left(2 m_{l}\right)}\right\rangle= \\
=i \times \text { real }
\end{gathered}
$$

is then purely imaginary, which implies that no contributions to the DoS, Eq. (7), obtain. Summarizing, we have found that the mere complex structure of the operators coupling to the DoS entails the absence of perturbative DoS corrections. Before moving on, let us make a few general remarks on the structure of the argument:

- The construction formulated above explicitly relies on the fact that the energy operator in the rotated representation is purely imaginary. At first sight it looks like this makes the derivation explicitly gauge dependent. Indeed, for order parameter phases $\phi \neq 0$ the rotation to the superconductor configuration introduces operators into the action that are neither purely real nor imaginary. However, it is straightforward to verify that (a) the phase couples only to the class of odd operators and (b) that any non-vanishing Wick contraction contains an equal number of phases $\exp (i \phi)$ and $\exp (-i \phi)$. This ensures the gauge invariance of the argument. 
- We emphasize that, in spite of the fact that the construction is based on the zero energy Eilenberger solution $g=\sigma_{2}$, it is by no means equivalent to a mere perturbative expansion of the DoS around zero energy. In fact we might have used any other low energy approximate solution $Q_{\mathrm{r}}$ - in particular, the trial solution, Eq. (3), may also have served as a reference configuration. As long as $Q_{\mathrm{r}}$ is not separated from the 'true' Eilenberger solution $\bar{Q}$ by non-analyticities, $\bar{Q}$ can be reached from $Q_{\mathrm{r}}$ by a perturbative expansion in $W$, and the conclusion of a vanishing DoS remains valid. The absence of non-analyticities for small energies is in turn guaranteed by the presence of the diffraction operator $\mathcal{O}_{\text {coll. }}$ As follows from the structure of the effective action, $\mathcal{O}_{\text {coll }}$ causes the induction of a stable BCS gap $\sim \hbar /(2 \tau)$ on all classical trajectories in the normal region. For subgap energies, $\epsilon \ll \hbar / \tau$, the mean field equations of the theory are regular and the perturbative scheme works. (The same is true for the regime of large energies $\epsilon \gg \hbar / \tau$ where the Bohr-Sommerfeld DoS of the non-diffractive system is retrieved.) In the vicinity of the gap region, however, the theory is fundamentally unstable and can not be used to produce reliable results.

- The stabilization of the gap by a non-analyticity in the functional integral can explicitly be tested for the particular case of diffusive SN systems. In the diffusive regime, the Eilenberger equation transmutes to the Usadel equation which, at least for a number of relevant geometries, can be solved in closed form (cf. the discussion of section II.) A field integral analysis of fluctuations the Usadel mean field solutions [10] then shows that no perturbative corrections to the zero subgap DoS exist. The responsible mechanism is perfectly analogous to the one discussed above.

We remark also, while we have demonstrated the absence of perturbative corrections to the zero subgap DoS, this conclusion does not rule out the possibility of contributions to the subgap DoS of a nonperturbative nature. For diffusive systems, such contributions to the subgap DoS do exist, as pointed out in Ref. [10] and later investigated in detail [38], and are associated with a rare class of states, known as 'tail' states. These states are poorly contacted with the superconductor and can exist below the gap to give rise to contributions to the DoS that are, for diffusive systems, exponentially small in the dimensionless conductance. In terms of the field theory, such states correspond to configurations of the $Q$-matrix that are perturbatively inaccessible from both the conventional Eilenberger saddle-point and the metallic saddle-point of the theory. (More precisely, such states can be associated with saddle points that break causality in the fermion-fermion sector of the theory.) The study of tail states in the ballistic regime, while an interesting and unexplored topic, is beyond the scope of the present work.

Finally, to prepare the analysis of soft scattering systems, we introduce a bridge between quasiclassics/field theory and the qualitative trajectory oriented pictures discussed in section [II. In order to keep the discussion simple, it is convenient to consider a situation where the non-diffractive Eilenberger solution is close to the metallic reference point (large energies) and can be interpreted as the quantum mechanical amplitude of pair propagation of ordinary electrons and holes. The extension to strongly proximity influenced regimes is technically straightforward but less easy to visualize.

The most basic constituent of the field theory discussed above is the propagator $\Pi$, i.e. the (inverse of the) kernel of the quadratic action. Expanding the action around the metallic reference configuration $Q=\sigma_{3}$ it is straightforward to show that in the diffractionless limit, the kernel is given by

$$
\Pi^{-1}=\frac{\pi \rho}{2}(-\hbar \mathcal{L}-2 i \epsilon)
$$

This means that the propagator of the theory in phase space, $\Pi\left(\mathbf{x}, \mathbf{x}^{\prime}\right)$ is just the $\epsilon$-Fourier transform of the classical time evolution operator,

$$
\begin{aligned}
\Pi\left(\mathbf{x}, \mathbf{x}^{\prime}\right)=\frac{2}{\pi \hbar \rho} \int_{0}^{\infty} & d t e^{2 i \epsilon t / \hbar}\left(e^{\mathcal{L} t}\right)\left(\mathbf{x}, \mathbf{x}^{\prime}\right)= \\
= & \frac{2}{\pi \hbar \rho} \int_{0}^{\infty} d t e^{2 i \epsilon t / \hbar} \delta\left(\mathbf{x}-\mathbf{x}^{\prime}(t)\right) .
\end{aligned}
$$

By expanding the functional integral in terms of the $B$ field, one may now describe the effect of Andreev scattering and/or quantum diffraction in terms of perturbative superpositions of classical propagators. For example, expanding the functional to lowest order around the quadratic kernel the first correction to the metallic DoS is found to be

$$
\delta \nu=-\pi \rho \Delta^{2} \nu_{0} \operatorname{Im} \frac{\partial}{\partial \epsilon} \int_{S} d \mathbf{x}_{1} d \mathbf{x}_{2} \Pi\left(\mathbf{x}_{1}, \mathbf{x}_{2}\right)
$$

where the $\Pi$ describes the propagation along the classical trajectory of an Andreev bound state (Fig. 0 , right), and $\int_{S}$ denotes an integration over the superconductor interface. 


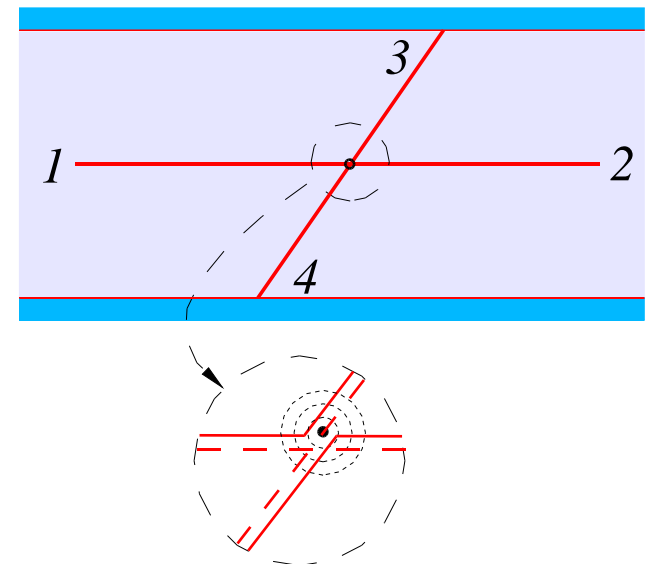

FIG. 6. First order quantum correction to the classical propagator in a SN-system with hard impurity scattering. Inset: solid line: propagation amplitude of an electron, dashed line: amplitude of the Andreev reflected hole state, dot: $s$-wave impurity scatterer.

In the same manner one verifies that, at next order in an expansion of the functional integral in the generators $B$, a quantum correction to the classical propagation of the type

$$
\begin{aligned}
& \delta \Pi\left(\mathbf{x}_{1}, \mathbf{x}_{2}\right)=-2 \frac{\hbar \Delta^{2}(\pi \rho)^{3}}{\tau} \int_{N} \frac{d \mathbf{r}}{V} \int \frac{d \mathbf{n}^{\prime} d \mathbf{n}}{S_{d}^{2}} \int_{S} d \mathbf{x}_{3} d \mathbf{x}_{4} \times \\
& \times \Pi\left(\mathbf{r}, \mathbf{n} ; \mathbf{x}_{1}\right) \Pi\left(\mathbf{x}_{3} ; \mathbf{r}, \mathbf{n}\right) \Pi\left(\mathbf{r}, \mathbf{n}^{\prime} ; \mathbf{x}_{4}\right) \Pi\left(\mathbf{x}_{2} ; \mathbf{r}, \mathbf{n}^{\prime}\right)+\ldots,
\end{aligned}
$$

appears. (Here the ellipses denote contributions of similar architecture but somewhat different structure in phase space.)

The integration $\int_{S}$ extends over (the phase space points on) the superconductor interface. A cartoon of this process is depicted in Fig. 6, where the inset represents a visualization of amplitudes involved in the diffractive $s$-wave scattering. We notice that $\delta \Pi$ represents the quantitative formulation (for a hard scattering ballistic environment) of the diffraction corrections discussed qualitatively in section II. Through such processes, an exceptionally long classical trajectory (quantitatively described by the propagator $\left.\Pi\left(\mathbf{x}, \mathbf{x}^{\prime}\right)\right)$ establishes contact with the superconductor on time scales $\tau$. Substitution of the modified propagator into the formula for $\delta \nu$ gives the first quantum correction to the DoS.

Technically, the quantum contribution to $\Pi$ can be interpreted as some 'self energy' correction of strength $\sim \hbar / \tau$. On small energy scales $\epsilon<\hbar / \tau$ the action of the diffraction operator is no longer perturbatively accessible. Nonetheless, the concept of a low order expansion around the limit of classical propagation remains a useful concept: In cases where the mechanism of quantum diffraction is less trivial than mere $s$-wave scattering, a perturbative analysis can be used to determine the characteristic energy scale beyond which the quantum propagation becomes dominated by diffractive mixing between classically isolated regions in phase space. The general structure of the theory implies that this time scale sets the width of the gap. We will come back to this point in the next section, when the soft scattering problem is discussed.

\section{CLEAN CHAOTIC SYSTEMS}

In this section we turn our attention to the proximity effect in clean chaotic systems. As before we focus on billiard type systems in which the average time of flight $t_{\mathrm{f}}$ represents the shortest time scale in the problem. We assume that the scattering potential is smooth. (In billiards, i.e. systems where the characteristic length scale of the scattering potential $a$ coincides with the system size, 'smooth' means $a \gg \lambda_{F}$. For a definition applicable under more general conditions, see [24].)

The traditional form of the Eilenberger equation, Eq. (1), applies to billiard type systems subject to weak impurity scattering. Its naive generalization to generic clean systems characterized by a set of phase space coordinates $\{\mathbf{x}\}$ is given by

$$
\left(\hbar \mathcal{L}-i\left[\sigma_{3}(\epsilon+\hat{\Delta}(\mathbf{x})),\right]\right) g(\mathbf{x})=0,
$$

with the standard non-linear normalization $g(\mathbf{x}) g(\mathbf{x})=$ $\mathbb{1}$. This equation differs from (11) by (i) the absence of an impurity scattering operator $\mathcal{O}_{\text {coll }}$ and (ii) the generalization from $v_{F} \mathbf{n} \cdot \partial_{\mathbf{r}}$ to an arbitrary Liouville operator.

A moment's thought, however, shows that Eq. (10) poses a serious problem: as discussed in section III, the diffractionless Eilenberger equation reproduces the BS result for the DoS. (No specific assumptions on the structure of the Liouville operator were made in that argument.) Relying on (10) one would thus have to conclude that the proximity DoS of clean chaotic systems is not gapped, i.e. that the DoS gap must be a peculiarity of disordered systems. This hypothesis, however, can be dismissed on the basis of a simple argument: being a positive definite quantity, the DoS of disordered systems can only vanish on average, if it vanishes for each individual realization. However, a given realization of a disordered system is nothing more than a Hamiltonian chaotic system. From this argument one expects that the proximity gap is a generic signature of chaotic dynamics (save, perhaps, for chaotic systems - unknown to us - that do not fall into the universality class of standard Hamiltonian systems with chaotic potential scattering.)

The resolution of the paradox is that for clean chaotic systems the derivation of (10) is ill-founded. Indeed, the derivation of the Eilenberger equation is based on the presumption that the quasiclassical Green functions varies slowly on the scale of the Fermi wave length. For disordered systems, the ensemble averaging over realizations makes sure that this requirement is met. With chaotic systems, however, the situation is different. The phase space eigenfunctions of the chaotic Liouville operator are singular and fluctuate on all length scales. This 
invalidates any semiclassical gradient expansion, unless a smoothing regularization mechanism is active.

Before discussing the issue of regularization further, we switch back to the language of the field integral formulation. The variational action corresponding to the clean Eilenberger equation is given by $S_{0}[T]$ of Eq. (4). After the rotation $T \rightarrow R T, R=\exp \left(i \pi \sigma_{1} / 4\right)$ to the superconductor reference point $\left(\bar{Q}=\sigma_{2}\right)$, the action simplifies to

$$
S_{0}[T]=-\pi \rho \int d \mathbf{x s t r}\left(\hbar T \sigma_{3} \mathcal{L} T^{-1}-i \epsilon \sigma_{2} Q\right)
$$

where coupling to the superconductor is implicit, through the boundary condition $T=\mathbb{1}$ at the SN-interface.

Within the context of the general ballistic $\sigma$-model, the necessity to regularize the effective action $S_{0}$ has been pointed out earlier 26, 39, 36]. More specifically, it has been noticed that the phase space singularity alluded to above invalidates the gradient expansion on which the derivation of the effective action $S_{0}$ is based. To give the effective action some meaning, a regularizing contribution $S_{\text {reg }}$ has to be added. Frustratingly, however, no generally accepted consensus regarding the concrete implementation of a regularization operator has been reached as yet. In fact, various different schemes have been proposed in the literature: in the early work [26] it was suggested to regularize the action by adding a second order differential operator of infinitesimal coupling strength. In Ref. 24] it was argued that a Hamiltonian chaotic system will self-generate some regularization if only the mechanism of quantum dispersion is consistently taken into account. In lack of a fully microscopic description of diffraction within a quasiclassical formalism, it was suggested to model phenomenologically the effect of the quantum spreading of minimal wave packets. This was done by adding the diffusion type second order operator

$$
S_{\mathrm{reg}}[Q]=-\frac{\hbar \pi \nu}{4 \tau_{\mathrm{r}}} \int d \mathbf{x} \operatorname{str}\left(\partial_{\mathbf{n}} Q \partial_{\mathbf{n}} Q\right)
$$

to the effective action, where the value of the characteristic scattering time $\tau_{\mathrm{r}}=\lambda^{2} v_{F} / \lambda_{F}$ and $\lambda$ is the largest Liapunuov exponent of the system (i.e. unlike in Ref. [26], the coupling strength of the regulator was kept finite.) Physically, the operator $S_{\mathrm{reg}}[Q]$ describes the effect of some soft (low momentum transfer) scattering off a stochastic potential; this may be seen readily by comparison with the collision term $S_{\text {coll }}$ in the action (四), in the case where $w\left(\mathbf{n}-\mathbf{n}^{\prime}\right)$ is strongly peaked around $\mathbf{n}-\mathbf{n}^{\prime} \sim 0$. We re-emphasize that this potential is fictitious and only serves to model the spreading inherent to the propagation of any quantum minimal wave package. In contrast, Ref. [39] argued that a chaotic system will not auto-regularize itself and that some external smoothing is needed. It was suggested to effect this regularization by adding to the action an elliptic second order differential operator coupled to some weak stochastic background field. Again in contradiction to Ref. [24], it was conjectured that a coupling strength vanishing in the classical limit would be sufficient to generate regularization.

Although no general consensus regarding the practical realization has been reached, it is clear that the ballistic $\sigma$-model needs regularization through some positive second order differential operator (or any other suitable scheme.) In view of this situation, we here take a pragmatic point of view and add to the action $S_{0}$ the contribution

$$
S_{\text {reg }} \equiv-\frac{\hbar \pi \rho D_{i j}}{4} \int d \mathbf{x} \operatorname{str}\left(\partial_{i} Q \partial_{j} Q\right)
$$

where $D_{i j}$ is a positive matrix whose characteristics will be left unspecified for the moment.

Before moving on it is perhaps worthwhile to reemphasize that this regularization problem is in no way particular to the field theoretical approach. It rather affects all semiclassical expansion schemes, including those underlying the derivation of the Eilenberger equation. In applications involving disorder, the averaging over a continuous set of potential variables effectively smoothens out all singularities, and the derivation of effective low energy theories is not met with difficulties. For individual representatives of chaotic systems, however, the problem is virulent and regularization must be an integral part of any quasiclassical approach. We re-emphasize that the actual mechanism by which regularization is effected 'in reality' is not well understood at present.

We now turn to the discussion of the effective action $S=S_{0}+S_{\text {reg }}$ determined by Eqs. (11) and (12), respectively. Comparison with (6) shows that $S$ bears much structural similarity with the action of the previously discussed $s$-wave scattering system. The essential difference lies in the structure of the scattering operators $S_{\text {coll }}$ and $S_{\text {reg }}$, respectively. Whilst $S_{\text {coll }}$ describes large momentum transfer scattering at a rate determined by $1 / \tau$, the diffractive scattering modelled by $S_{\text {reg }}$ is soft and of a strength determined by the matrix $D$. Naively, one might conjecture that this difference in the microscopic structure cannot be of much concern and that $S_{\text {reg }}$ acts qualitatively similar to $S_{\text {coll }}$. This anticipation is wrong. In contrast to $S_{\text {coll }}$, the operator $S_{\text {reg }}$ leads to a formation of a gap only for systems with underlying chaotic dynamics. Further, the width of that gap depends only logarithmically on the coupling strength, $D$.

To understand the action of the diffraction operator we consider the soft scattering analogue of the perturbative picture developed in the end of the previous section. Expanding the action to second order in the generators $B$, it is straightforward to verify that, in the presence of soft diffraction, the classical propagator (8) generalizes to

$$
\Pi^{-1}=\frac{\pi \rho}{2}\left(-\hbar \mathcal{L}-2 i \epsilon-\hbar D_{i j} \partial_{i} \partial_{j}\right) .
$$

One further verifies that the first order quantum correction to the propagation along a classical trajectory is given by 


$$
\begin{aligned}
\delta \Pi\left(\mathbf{x}_{1}, \mathbf{x}_{2}\right)= & 8(\pi \rho)^{3} \Delta^{2} \hbar D_{i j} \int d \mathbf{x} \int_{S} d \mathbf{x}_{3} d \mathbf{x}_{4} \times \\
& \times \partial_{\mathbf{x}_{i}} \Pi\left(\mathbf{x}, \mathbf{x}_{1}\right) \Pi\left(\mathbf{x}_{3}, \mathbf{x}\right) \Pi\left(\mathbf{x}, \mathbf{x}_{4}\right) \partial_{\mathbf{x}_{j}} \Pi\left(\mathbf{x}_{2}, \mathbf{x}\right)
\end{aligned}
$$

(compare with expression (9) for $\delta \Pi$ in the case of hard, $s$-wave scattering). At first sight, the structure of this expression suggests that upon sending the regulator strength to zero, the quantum corrections to classical propagation vanish linearly with $D$. However, this anticipation is too naive; it does not account for potentially singular behavior of the $\mathbf{x}$-derivatives acting on the propagator $\Pi$.

To better understand the behavior of the quantum correction for small $D$, we need to explore the structure of the regularized propagator $\Pi$. Consider the time representation of the defining equation of $\Pi$,

$$
\frac{\pi \rho}{2}\left(d_{t}-\mathcal{L}_{\mathbf{x}}-D_{i j} \partial_{x_{i}} \partial_{x_{j}}\right) \Pi\left(\mathbf{x}, \mathbf{x}^{\prime} ; t\right)=\delta\left(\mathbf{x}-\mathbf{x}^{\prime}\right) \delta(t),
$$

where $t \geq 0$ and the subscript in $\mathcal{L}_{\mathbf{x}}$ indicates that the Liouvillian acts on the argument $\mathbf{x}$. For notational convenience, the factor of two multiplying the energy argument $\epsilon$ has been included in the definition of the time Fourier argument $t$. In the absence of diffraction, $\Pi_{D=0}\left(\mathbf{x}, \mathbf{x}^{\prime} ; t\right)=(2 / \pi \rho) \delta\left(\mathbf{x}-\mathbf{x}^{\prime}(t)\right)$. For finite $D_{i j}, \Pi$ is no longer purely deterministic but rather describes a stochastic process of diffusion centered around the classical trajectory $\mathbf{x}^{\prime}(t)$. For sufficiently small times, the argument $\mathbf{x}$ is close to $\mathbf{x}^{\prime}(t)$ and the equation for $\Pi$ can be linearized in $\mathbf{y} \equiv \mathbf{x}-\mathbf{x}^{\prime}(t)$. Introducing $\mathcal{L} \equiv I_{i j} \partial_{x_{j}} H \partial_{x_{i}}$, where the matrix

$$
I=\left(\begin{array}{ll} 
& \mathbb{1} \\
-\mathbb{1} &
\end{array}\right)
$$

implements the symplectic structure of the phase space, the linearized equation for $\Pi$ assumes the form

$$
\begin{aligned}
\left(d_{t}\right. & \left.-\mathcal{L}_{\mathbf{x}^{\prime}(t)}-M_{i j} \partial_{y_{i}} y_{j}-D_{i j} \partial_{y_{i}} \partial_{y_{j}}\right) \Pi\left(\mathbf{x}^{\prime}(t)+\mathbf{y}, \mathbf{x}^{\prime} ; t\right) \\
& =\frac{2}{\pi \rho} \delta(\mathbf{y}) \delta(t)
\end{aligned}
$$

with $\left.M_{i j} \equiv I_{i k} \partial_{x_{j} x_{k}}^{2} H\right|_{\mathbf{x}=\mathbf{x}^{\prime}(t)}$. Noticing that for any function $f\left(\mathbf{x}^{\prime}(t)\right)$ that is not explicitly dependent on $t$, we have $\left(d_{t}-\mathcal{L}_{\mathbf{x}^{\prime}(t)}\right) f\left(\mathbf{x}^{\prime}(t)\right)=0$, the differential equation can be rewritten as

$$
\left(d_{t}-M_{i j} y_{j} \partial_{y_{i}}-D_{i j} \partial_{y_{j}} \partial_{y_{i}}\right) \Pi(\mathbf{y} ; t)=\frac{2}{\pi \rho} \delta(\mathbf{y}) \delta(t),
$$

where the dependence on both $\mathbf{x}^{\prime}(t)$ and the initial condition are implicit, and the time derivative acts only on the explicit time argument in $\Pi(\mathbf{y} ; t)$. Eq. (15) is a differential equation of Fokker-Planck type. Owing to the complex time dependence of the matrix $M$ it is, of course, not analytically solvable. Nonetheless, some qualitative statements regarding the structure of the solution can be made: Eq. (15) is governed by a competition between the diffusion operator $D_{i j} \partial_{i} \partial_{j}$, and the drift operator $M_{i j} \partial_{y_{i}} y_{j}$. By definition, the time averaged eigenvalues of the 'drift matrix' $M$ are the Liapunov exponents of the chaotic scattering problem. On short time scales, the dynamics of the equation is controlled by the diffusion term and we expect the distribution $\Pi$ to describe a stochastic diffusive spreading around the deterministic solution $\mathbf{x}^{\prime}$. After a certain threshold time, a crossover to a regime controlled by the drift operator takes place. On larger time scales, the distribution $\Pi$ describes some exponential separation away from the trajectory $\mathbf{x}^{\prime}$, where the rate of separation is determined by the Liapunov exponents.

To formulate these concepts in a somewhat more quantitative manner we make the assumption (acceptable on short time scales) that the matrix $M$ is approximately static. (In fact, all what we really need to require is that the largest Liapunov exponent, $\lambda$, and the associated direction in phase space are constant.) For a time independent matrix $M$, Eq. (15) can be solved in closed form 40]:

$$
\Pi(\mathbf{y}, t)=\frac{4}{\rho(2 \pi)^{(2 d+1) / 2}}(\operatorname{det} \sigma(t))^{-1 / 2} e^{-\frac{1}{2} \mathbf{y}^{T} \sigma(t)^{-1} \mathbf{y}},
$$

where the matrix-kernel $\sigma$ is given by

$$
\sigma(t)=2 \sum_{\alpha \beta} \frac{e^{\left(\lambda_{\alpha}+\lambda_{\beta}\right) t}-1}{\lambda_{\alpha}+\lambda_{\beta}} \mathbf{v}^{\alpha}\left(\mathbf{v}^{\alpha T} \mathbf{D} \mathbf{v}^{\beta}\right) \mathbf{v}^{\beta T} .
$$

Here $\mathbf{v}^{\alpha}$ and $\lambda_{\alpha}$ are eigenvectors and eigenvalues of the drift matrix $M$, respectively. The enumeration of the eigenvalues is ordered such that $\lambda \equiv \lambda_{0}>\lambda_{1}>\ldots>$ $\lambda_{2 d-1}$. Finally, re-installing our original set of variables, we arrive at

$$
\begin{aligned}
\Pi\left(\mathbf{x}, \mathbf{x}^{\prime}, t\right)=\frac{4}{\rho(2 \pi)^{(2 d+1) / 2}}(\operatorname{det} \sigma(t))^{-1 / 2} \times \\
\times e^{-\frac{1}{2}\left(\mathbf{x}-\mathbf{x}^{\prime}(t)\right)^{T} \sigma(t)^{-1}\left(\mathbf{x}-\mathbf{x}^{\prime}(t)\right)}
\end{aligned}
$$

for the short time approximation to the propagator $\Pi$. From this expression we recover the temporal picture discussed qualitatively above: For times shorter than $\lambda^{-1}$, $\sigma \approx D t$ and the solution describes diffusion around the classical trajectory $\mathbf{x}^{\prime}$. On time scales larger than $\lambda^{-1}$, some of the matrix elements of $\sigma$ begin to diverge exponentially. Defining $x_{0}=\mathbf{v}^{0 T} \mathbf{x}$ as the projection of $\mathbf{x}$ onto the direction of strongest exponential divergence, we find

$$
\left\langle\left(x_{0}-x_{0}^{\prime}(t)\right)^{2}\right\rangle \stackrel{t \gg \lambda^{-1}}{\approx} \frac{D_{0}}{\lambda} e^{\lambda t}
$$

where $D_{0} \equiv \mathbf{v}^{0 T} D \mathbf{v}^{0}$ denotes the relevant element of the diffusion matrix. Thus, for $t>\lambda^{-1}$ the propagation is no longer confined to the vicinity of $\mathbf{x}^{\prime}(t)$ but rather branches out exponentially, at a rate determined by the largest Liapunov exponent of the system. We emphasize that this type of behavior is specific to chaotic systems. 
For an integrable system, no globally positive exponents driving the separation process exist, and, the weak diffusive smearing left aside, $\Pi$ does not deviate from the predestined trajectory $\mathbf{x}^{\prime}$.

We now turn back to our discussion of the quantum correction, $\delta \Pi$ of Eq. (13). Specifically we wish to understand how much time it takes before an exceptionally long classical trajectory diffractively spreads out into the phase space regions strongly coupled to the superconductor. The first thing to be observed is that the prefactorial dependence of $\delta \Pi$ in Eq. (13) on the diffusion matrix $D_{i j}$ is largely cancelled by the derivatives acting on the propagators $\Pi$. Indeed, the diffraction correction at fixed energy, $\epsilon$, is determined by the time Fourier transform of the propagators $\Pi\left(\mathbf{x}, \mathbf{x}^{\prime} ; t\right)$. Parametrically, the contribution of short times $t<\lambda_{\alpha}^{-1}$ to the time Fourier integral of $\partial_{\mathbf{x}} \Pi$ is given by

$$
\partial_{\mathbf{x}} \Pi \sim\left(\mathbf{x}-\mathbf{x}^{\prime}\right) \sigma^{-1} \Pi \sim \sigma^{-1 / 2} \Pi \stackrel{t \ll \lambda_{\alpha}^{-1}}{\sim}(D t)^{-1 / 2} \Pi,
$$

implying that the $D$-dependence introduced by the two derivatives cancels against that of the prefactor. From this rough estimate we conclude that it will not be the naive timescale related to the coupling strength of the operator that sets the effective rate of diffraction, but rather some other time scale.

To determine that scale we apply a modified version of an argument first formulated by AL within the context of normal metal weak localization: let us focus attention on the coordinate $x_{0}$ of strongest exponential divergence. For the sake of concreteness, we make the (inessential) assumption that $x_{0}$ represents some real space coordinate. From the solution for $\Pi$ we find that for short time scales $t<\lambda^{-1},\left\langle\left(x_{0}-x_{0}^{\prime}(t)\right)^{2}\right\rangle \approx D_{0} t$. On the other hand, the dispersive quantum spreading of a Gaussian minimal wave packet centered around the classical trajectory would be estimated by $\left\langle\left(x_{0}-x_{0}^{\prime}(t)\right)^{2}\right\rangle \sim v_{F} \lambda_{F} t$. We now follow the general philosophy of AL and postulate that the mere function of the regularization operator is to phenomenologically mimic this effect. This leads to the estimate $D_{0} \sim v_{F} \lambda_{F}$ [11].

Having parametrically estimated the strength of the diffraction operator, we next consider the quantum correction, $\delta \Pi$ of Eq. (13), in the 'Liapunov regime' of time scales $t>\lambda^{-1}$. To couple a formerly isolated exceptionally long trajectory $\mathbf{x}^{\prime}: \mathbf{x}_{1} \rightarrow \mathbf{x}_{2}$ to the background of average trajectories and/or the superconductor interface, the propagators $\Pi\left(\mathbf{x}_{3}, \mathbf{x}\right)$ and $\Pi\left(\mathbf{x}, \mathbf{x}_{4}\right)$ 'branching out' from the point $\mathbf{x}$ of diffractive scattering, have to leave the immediate phase space vicinity of the reference trajectory. A conservative estimate of the thresholddeviation necessary to initiate that coupling reads as

$$
\left\langle\left(x_{0}-x_{0}^{\prime}(t)\right)^{2}\right\rangle \approx \frac{D_{0}}{\lambda} e^{\lambda t} \sim a^{2},
$$

i.e. we demand that the spreading reaches a scale comparable with the system geometry. Finally, estimating the
Liapunov exponent as $\lambda \sim v_{F} / a$, and using the above expression for $D_{0}$, we arrive at

$$
t \approx \frac{1}{\lambda} \ln \left(\frac{a}{\lambda_{F}}\right),
$$

which is the celebrated Ehrenfest time $t_{\mathrm{E}}$.

Summarizing we have found that for chaotic systems with smooth potentials, $t_{\mathrm{E}}$ is the time scale at which quantum corrections begin to significantly affect the classical propagation along ballistic trajectories. On time scales larger than $t_{\mathrm{E}}$ any formerly isolated long classical trajectory 'merges' into the background of surrounding trajectories of average flight time $t_{\mathrm{f}}$ (where $t_{f}$ is the escape time). According to the general structure of the theory, the inverse of this threshold time, $\hbar / t_{\mathrm{E}}$, sets the width of the DoS gap.

It is instructive to study this mechanism more explicitly on the basis of the Eilenberger equation. This is done in appendix $B$, where we consider the prototypical example of an infinitely long trajectory $\mathbf{x}^{\prime}$ that, classically, would never hit the superconductor interface. In the absence of diffraction, the Green function along that trajectory is metallic, $g\left(\mathbf{x}^{\prime}, \epsilon\right)=\sigma_{3}$, and gives a finite contribution to the DoS. For $\mathcal{O}_{\text {coll }}$ weak but finite, the behavior of $g$ close to $\mathbf{x}^{\prime}$ is described by a Fokker-Planck equation similar to the one discussed above. The solution of this equation, detailed in Appendix B, shows that for energies $\epsilon \sim \hbar / t_{E}$, a crossover from $\sigma_{3}$ to the off-diagonal configuration set by the surrounding superconductor takes place. For still smaller energies the DoS vanishes.

Before concluding, let us make some general critical remarks on the status of the results obtained in this section. Unlike in the case of hard point-like scatterers, the derivation and solution of quasiclassical equations (alias the mean field equation of the ballistic $\sigma$-model) relied on various phenomenological assumptions:

- A number of assumptions regarding the nature of the Liapunov exponents of the system, and the solution of the linearized equations of motion have been made. Furthermore, some relevant classical and quantum system parameters have been estimated in a crude manner.

- The all-important diffraction operator has not been microscopically derived but was introduced on phenomenological grounds.

We believe that the first of these points is not serious. The reason is that the characteristic energy scale $\hbar / t_{\mathrm{E}}$ depends only logarithmically on the modelling of the dynamics, and on the values assigned to the parameters $D, \lambda, a$, etc. As long as a globally positive Liapunov exponent exists, an energy scale of the order of $\hbar / t_{\mathrm{E}}$ will be generated. The second point, however, is more important. The absence of a consistent microscopic derivation of a diffraction operator indeed represents a deficiency of the current approach and, for that matter, of 
the general field theory approach to quantum chaos. On the other hand, the presence of a diffraction operator, of the coupling strength specified above, is required for basic physical reasons. We therefore believe that, at least phenomenologically, the current approach stands on solid ground.

Finally, we notice that, unlike in the case of the hard scattering problem, it was not possible to bring the Eilenberger equation into a form manifestly equivalent to a bulk BCS equation with induced order parameter. This implies that the current approach cannot rigorously establish the existence of a hard gap edge for the soft scattering problem. What we could see is that on energy scales of the order of the inverse of the Ehrenfest time, a quantum mechanism suppressing the DoS becomes operative. The fact that for lower energies the solutions of the Eilenberger equation undergo a rotation towards the bulk superconductor configuration (cf. Appendix B) implies that the BS approach qualitatively overestimates the low energy DoS of chaotic systems.

\section{SUMMARY}

In this paper we have discussed various physical mechanisms relevant to the phenomenology of the proximity effect in chaotic SN-systems. Considering systems where chaotic dynamics is induced by a weak amount of point-like impurities as a test case, we have demonstrated that (a) the existence of a hard gap in the DoS is directly related to an essential singularity in the quasiclassical equations for the Green function and (b) that these singularities can be interpreted semiclassically as a non-perturbative accumulation of quantum interference processes akin to weak localization corrections in normal metals. These contributions are effectively summed up in the solutions of the quasiclassical equations. Although closed solutions can be obtained only in exceptional cases (for example in the excessively investigated case of systems with diffusive dynamics) we have shown that the strict vanishing of the DoS for low energies follows from combining elements of quasiclassics with the formalism of the ballistic $\sigma$-model. While all perturbative corrections to the DoS vanish below the gap, we have remarked that exponentially small contributions due to tail states are not ruled out by our analysis, but remain beyond the scope of this work.

Turning to the far more complex case of clean chaotic systems we have discussed various difficulties obstructing the construction of quantitative analytical approaches. The perhaps most fundamental problem is that the assumption of a spatially continuous Green function underlying, for example, the quasiclassical formalism fails (due to the singular distribution of the phase space eigenfunctions of the Liouville operator.) In principle one may evade this difficulty by averaging the theory over some set of external parameters. Structure and strength of a minimum set of averaging parameters needed to ensure continuity are not known at present. However, taking a pragmatic point of view and assuming continuity of the Green function, it can be demonstrated that for energy scales smaller than the inverse of the Ehrenfest time, quantum diffraction mechanisms become operative 24]. For those low energies the DoS of SN systems is suppressed below the value predicted by the semiclassical Bohr-Sommerfeld approach. (Here it is assumed that the Ehrenfest time is larger than the average classical escape time from the normal metal region. The opposite regime has not been investigated so far.) Most likely, the inverse of the Ehrenfest time marks the position of a hard gap edge although, due to the problem stated above and other difficulties related to the formulation of quantitative theories of chaotic systems (see the text), this could not be rigorously shown. In consideration of the difficulties mentioned above, and the fact that established semiclassical techniques based on counting classical trajectories fall short of producing a hard gap, a quantitative theory of the proximity effect remains a challenge for future approaches to chaotic sytems.

\section{ACKNOWLEDGMENTS}

We are grateful to O. Agam, M. Janssen and Yu. Nazarov for useful discussion during the course of this work and gratefully acknowledge the financial support of the 'Sonderforschungsbereich' 237.

\section{APPENDIX A: DERIVATION OF THE BALLISTIC SIGMA-MODEL}

In this section we derive the effective action (14) of a weakly disordered sample in the presence of a superconducting order parameter. While, as discussed in the main text, an action of this type for a normal metal was first proposed by Muzykantskii and Khmelnitskii [25], we follow here more closely the derivation of Andreev et al. [26], with suitable modifications: we allow for the presence of a superconducting order parameter by introducing particle and hole components in the functional integral. The method relies on an averaging over a set $N \gg 1$ of energy levels around the Fermi surface to construct the $\sigma$-model in the absence of disorder. The derivation employs further procedures that are central also to the quasiclassical approximation, including a Wigner-transform and integration over the momentum coordinate normal to the energy shell. The disorder is treated as a perturbation to derive a term resembling a collision integral in the action. Since we present the generating functional for a single retarded Green function, the role of the usual advancedretarded components in the field integral are taken over by particle-hole components. For simplicity we restrict ourselves to the case of broken time-reversal symmetry, 
although this simplification is not essential and the generalization to other symmetry classes is straightforward.

The starting point is the Gorkov Hamiltonian,

$$
\hat{H}=\hat{H}_{0}+V(\mathbf{r})+\hat{\Delta} \sigma_{3} ; \quad \hat{H}_{0}=\frac{\hat{\mathbf{p}}^{2}}{2 m} .
$$

The generating function for the single-particle Green function may be expressed as a field integral in the form

$$
\begin{aligned}
Z[\hat{J}]= & \int D \Psi \times \\
& \times \exp \left[-i \int d \mathbf{r} \Psi^{\dagger}(\mathbf{r}) L\left(\hat{G}^{-1}\left(\epsilon_{0}\right)+i \hat{J} E_{11}^{\mathrm{BF}}\right) \Psi(\mathbf{r})\right]
\end{aligned}
$$

where $\hat{G}^{-1}\left(\epsilon_{0}\right)=\epsilon_{0}-\epsilon \sigma_{3}-\hat{H} . \quad \Psi$ represents a four-component superfield, containing particle-hole and boson-fermion sectors. The energy $\epsilon$ represents an energy difference between particle and hole sectors. The matrix $L$ is defined as

$$
L=\left(\begin{array}{cc}
1 & \\
& \sigma_{3}^{\mathrm{BF}}
\end{array}\right),
$$

with the block decomposition being in particle-hole space. Two dimensional matrix field $\hat{J}=\left\{J_{a b}(\mathbf{x})\right\}$ serves as a source field to generate correlation functions, and $E_{11}^{\mathrm{BF}}$ is defined as $\left(E_{11}^{\mathrm{BF}}\right)_{i j}=\delta_{i 1} \delta_{j 1}$. Next we employ Gaussian averaging over energy levels according to

$$
\langle\ldots\rangle_{\epsilon_{0}}=\int \frac{d \epsilon_{0}}{\left(2 \pi N^{2}\right)^{1 / 2}} \exp \left[-\left(\epsilon_{0}-\epsilon_{F}\right)^{2} /\left(2 N^{2}\right)\right] .
$$

This induces a nonlocal interaction term in the generating functional:

$$
\begin{aligned}
\langle Z(\hat{J})\rangle_{\epsilon}= & \int D \Psi \exp \left[-i \int d \mathbf{r} \Psi^{\dagger}(\mathbf{r}) L \times\right. \\
& \left.\times\left(\hat{G}^{-1}\left(\epsilon_{F}\right)+i \hat{J} E_{11}^{\mathrm{BF}}\right) \Psi(\mathbf{r})-S_{\mathrm{int}}[\Psi]\right], \\
S_{\mathrm{int}}= & \frac{N^{2}}{2}\left(\int d \mathbf{r} \Psi^{\dagger}(\mathbf{r}) L \Psi(\mathbf{r})\right)^{2} .
\end{aligned}
$$

The interaction term is then decoupled with a matrix $Q\left(\mathbf{r}, \mathbf{r}^{\prime}\right)$ that is nonlocal in space: after integrating over $\Psi$ we find

$$
\begin{aligned}
& \langle Z(\hat{J})\rangle_{\epsilon_{0}}=\int D Q \exp \left[\frac{1}{2} \operatorname{str}_{\mathbf{r}} Q^{2}\right. \\
& \left.-\operatorname{str}_{\mathbf{r}} \ln \left(\hat{G}_{0}^{-1}(\hat{Q})-\sigma_{3}(\epsilon+\hat{\Delta})-V+i \hat{J} E_{11}^{\mathrm{BF}}\right)\right],
\end{aligned}
$$

where $\hat{G}_{0}^{-1}=\epsilon_{F}-\hat{H}_{0}-i N Q$ and $Q$ obeys the symmetry $Q^{\dagger}=L Q L$, where the dagger denotes a Hermitian conjugate. The strategy now is to simplify the action so as to isolate fluctuations associated with energies much less than the Fermi energy. To do so we search for a value of $Q$ that minimizes the action and expand in fluctuations around this saddle-point. In addition, we consider first the totally clean case $(V(\mathbf{r})=0)$, while later we include the effects of ballistic disorder. The saddle-point equation now reads as

$$
Q_{0} G_{0}^{-1}\left(Q_{0}\right)=-i N,
$$

with the explicit solution

$$
Q_{0}=-i \frac{\epsilon_{F}-\hat{H}_{0}}{2 N}+\left(1-\left(\frac{\epsilon_{F}-\hat{H}_{0}}{2 N}\right)^{2}\right)^{1 / 2} \sigma_{3} .
$$

To describe the low-energy, long-wavelength fluctuations of $Q$ around the saddle-point [42], we may write $Q=$ $T Q_{0} T^{-1}$, where $T$ obeys the symmetry $T^{\dagger}=L T L$, and expand the action to leading order in $1 / N$ : we find

$$
S[Q]=-\frac{i}{N} \operatorname{str}\left[Q\left(\sigma_{3}(\epsilon+\hat{\Delta})-i \hat{J} E_{11}^{\mathrm{BF}}-T^{-1}\left[\hat{H}_{0}, T\right]\right)\right] .
$$

At energies far below the Fermi energy, the leading contribution to the above functional integral may be described by the semiclassical approximation (note that the use of the term 'semiclassical' has an entirely different meaning from that used in the BS approach; here it stands for an expansion of operator traces in powers of $\hbar)$. To define this approximation, we apply the Wigner transformation

$$
\mathcal{O}(\mathbf{R}, \mathbf{p})=\int d(\delta \mathbf{r}) e^{i \mathbf{p} . \delta \mathbf{r} / \hbar} \mathcal{O}\left(\mathbf{R}+\frac{\delta \mathbf{r}}{2}, \mathbf{R}-\frac{\delta \mathbf{r}}{2}\right) .
$$

According to the semiclassical approximation, the Wigner transform of a product of operators may then be approximated as $\left(\mathcal{O}_{1} \mathcal{O}_{2}\right)(\mathbf{r}, \mathbf{p})=\mathcal{O}_{1}(\mathbf{r}, \mathbf{p}) \mathcal{O}_{2}(\mathbf{r}, \mathbf{p})+$ $\hbar\left\{\mathcal{O}_{1}(\mathbf{r}, \mathbf{p}), \mathcal{O}_{2}(\mathbf{r}, \mathbf{p})\right\}+\ldots$, where $\{\cdot, \cdot\}$ denotes the classical Poisson bracket. Specifically, the semiclassical approximation of the commutator with the Hamiltonian reads as

$$
\left[\hat{H}_{0}, T\right] \rightarrow-i \hbar \mathcal{L} T=-i \hbar\left\{T, H_{0}\right\},
$$

where $\mathcal{L}$ is the Liouville operator. Further, assuming that (a) fluctuations transverse to the constant energy shell $H_{0}=$ const. are frozen out and (b) $Q_{0} \approx \sigma_{3}$ (see Ref. [36] for a discussion of these points) we may write the semiclassical approximation of the matrices $Q$ as

$$
Q(\mathbf{x})=T(\mathbf{x}) \sigma_{3} T(\mathbf{x})^{-1},
$$

where $\mathbf{x}$ is a shorthand notation for the $2 d$-1-dimensional set of phase space coordinates of the constant energy shell. For a billiard type system with hard boundary scattering $\mathbf{x}=(\mathbf{r}, \mathbf{n})$.

Performing the integration over the coordinate transverse to the energy shell (for constant $Q$ ), we obtain from Eq. A2 the $S_{0}$ contribution to the action contained in Eq. (41). This part of the action is applicable to a clean sample in the presence of energy averaging. 
To include the effects of ballistic disorder, we may now reintroduce the impurity potential $V(\mathbf{r})$. Although for short range impurities this part of the Hamiltonian may not be treated by the semiclassical approximation, in the limit of weak disorder it may be treated as a small perturbation in a similar manner to the symmetry breaking terms $\hat{J} E_{11}^{\mathrm{BF}}$ and $\epsilon \sigma_{3}$ appearing in the action (A1). Expanding the logarithm to second order in $V$ and averaging over impurity configurations then leads to the collision term $S_{\text {coll }}$ in Eq. (4).

It may be verified (see main text) that the saddle-point equation for $\bar{Q}$ that minimizes the effective action (4) is precisely the the quasiclassical Eilenberger equation, Eq. (1). The strength of the field theory approach however is that it enables evaluation of the single-particle, impurity-averaged Gorkov Green function even beyond the quasiclassical approximation. Differentiation of the partition function with respect to the source term, $\hat{J}$, leads to

$$
\begin{aligned}
G(\mathbf{r}, \mathbf{n}) & =\left.\frac{1}{\pi \nu_{0}} \frac{\partial Z[\hat{J}]}{\partial \hat{J}(\mathbf{r}, \mathbf{n})}\right|_{\hat{J}=0} \\
& =\int D Q e^{-S[Q]} Q_{11}(\mathbf{r}, \mathbf{n})
\end{aligned}
$$

where $G(\mathbf{r}, \mathbf{n})$ is the Wigner-transformed and energyintegrated form of the Gorkov Green function, and the 11 indices refer to $\mathrm{BF}$ space. While evaluation of the integral in Eq. A3) at the saddle-point level reproduces the quasiclassical approximation for $G(\mathbf{r}, \mathbf{n}) \simeq \bar{Q}(\mathbf{r}, \mathbf{n})$, integration over the entire $Q$-manifold in principle reproduces the exact expression for $G(\mathbf{r}, \mathbf{n})$.

\section{APPENDIX B: APPEARANCE OF THE EHRENFEST TIME WITHIN THE QUASICLASSICAL FORMULATION}

In this appendix we explore the influence of the collision operator on the solution of the Eilenberger equation in a chaotic environment. Specifically, we consider the equation in the vicinity of a classical trajectory $\mathbf{x}^{\prime}$ of infinite length, i.e. a trajectory that never hits the superconductor interface. This trajectory is meant to serve as a caricature of the long trajectories that support the BS density of states at low energies.

As long as the collision operator is not included, the quasiclassical Green function on our trajectory assumes its metallic value, $g\left(\mathbf{x}^{\prime}, \epsilon\right)=\sigma_{3}$. For coordinates $\mathbf{x}$ away from the reference trajectory and/or in the presence of a finite collision term we expect deviations from $\sigma_{3}$. To parameterize these configurations we introduce the parameterization $g(\mathbf{x})=e^{W(\mathbf{x})} \sigma_{3} e^{-W(\mathbf{x})}$, where $W$ is a $2 \times 2$ antiunitary matrix generating rotations around $\sigma_{3}$. For all what follows it will be convenient to parameterize $W$ as $W=W_{+}+W_{-}$, where

$$
W_{ \pm}=i w_{ \pm} \frac{1}{\sqrt{2}}\left(\sigma_{1} \mp i \sigma_{2}\right), \quad w_{ \pm} \in \mathcal{R} .
$$

Anticipating that in the vicinity of $\mathbf{x}^{\prime}, W$ will be small, we next expand $g$ as $g=\sigma_{3}+2 W \sigma_{3}+\ldots$ and linearize the Eilenberger equation

$$
\left(\hbar \mathcal{L}-i \epsilon\left[\sigma_{3}, \quad\right]\right) g(\mathbf{x})-\hbar D_{i j} \partial_{i}\left(g(\mathbf{x}) \partial_{j} g(\mathbf{x})\right)=0,
$$

in $W$. The result reads as

$$
\left( \pm \hbar \mathcal{L}+2 i \epsilon+\hbar D_{i j} \partial_{i j}^{2}\right) w_{ \pm}=0
$$

To make progress with this equation, it is again convenient to transform to time space. Fourier transforming we obtain

$$
\left( \pm \mathcal{L}-d_{t}+D_{i j} \partial_{i j}^{2}\right) w_{ \pm}=0
$$

where, as in the main text, the factor of 2 multiplying the energy argument $\epsilon$ has been included in the definition of $t$. To give this equation some meaning, initial conditions have to be specified. To this end we introduce a coordinate system where the first of the $2 f$ phase space coordinates $y, y_{0}, y_{1}, \ldots, y_{2 d-1}$ parameterizes our reference trajectory, whereas the remaining coordinates span the complementary region. We next assume than all possible choices of initial conditions $w_{ \pm}(\mathbf{x}, t=0) \equiv w_{ \pm, 0}(\mathbf{x})$ have the common feature that $w_{ \pm, 0}$ increases up to $w_{ \pm}=\mathcal{O}(1)$ as any of the coordinates $x_{0}, \ldots, x_{2 d-1}$ approaches the characteristic scale of the potential, $a$. What this means is that for phase space points farther away from the exceptional trajectory than $a$, the Green function is locked to the superconductor order parameter. We notice that neither the specific choice of the threshold value $x_{i} \approx a$ nor the rigorous implementation of the initial condition sensitively affect the results of our analysis; it suffices that $w_{ \pm, 0}\left(x_{i} \sim a\right) \sim 1$ holds in some average sense.

The formal solution of our initial condition problem now reads as

$$
w_{ \pm}(\mathbf{x}, t)=\int d \mathbf{x}^{\prime} K_{ \pm}(\mathbf{x}, \overline{\mathbf{x}}, t) w_{ \pm, 0}(\overline{\mathbf{x}})
$$

where the kernel $K$ is defined through

$$
\left( \pm \mathcal{L}-d_{t}+D_{i j} \partial_{i j}^{2}\right) K_{ \pm}(\mathbf{x}, \overline{\mathbf{x}}, t)=\delta(t) \delta(\mathbf{x}-\overline{\mathbf{x}})
$$

This equation is equivalent to Eq. (14) for the propagator $\Pi$ and we can directly transcribe the solution. Substituting

$$
\begin{aligned}
K_{ \pm}(\mathbf{x}-\overline{\mathbf{x}}, t)= & \mp(2 \pi)^{-(2 d-1) / 2}\left(\operatorname{det} \sigma_{ \pm}\right)^{-1 / 2} \times \\
& \times e^{-\frac{1}{2}(\mathbf{x}-\overline{\mathbf{x}}(t)) \sigma_{ \pm}^{-1}(\mathbf{x}-\overline{\mathbf{x}}(t))}
\end{aligned}
$$

with

$$
\sigma_{ \pm}(t)= \pm 2 \sum_{\alpha \beta} \frac{e^{ \pm\left(\lambda_{\alpha}+\lambda_{\beta}\right) t}-1}{\lambda_{\alpha}+\lambda_{\beta}} \mathbf{v}^{\alpha}\left(\mathbf{v}^{\alpha T} \mathbf{D} \mathbf{v}^{\beta}\right) \mathbf{v}^{\beta T}
$$

into the integral representation for $w_{ \pm}$, one obtains 


$$
\begin{aligned}
& w_{ \pm}(\mathbf{x}, t)= \\
& =\mp(2 \pi)^{-(2 d-1) / 2}\left(\operatorname{det} \sigma_{ \pm}\right)^{-1 / 2} \times \\
& \times \int d \mathbf{x} e^{-\frac{1}{2}(\mathbf{x}-\overline{\mathbf{x}}(t)) \sigma_{ \pm}^{-1}(\mathbf{x}-\overline{\mathbf{x}}(t))} w_{ \pm, 0}(\overline{\mathbf{x}}) \\
& =\mp(2 \pi)^{-(2 d-1) / 2}\left(\operatorname{det} \sigma_{ \pm}\right)^{-1 / 2} \times \\
& \times \int d \mathbf{x} e^{-\frac{1}{2}(\mathbf{x}-\overline{\mathbf{x}}) \sigma_{ \pm}^{-1}(\mathbf{x}-\overline{\mathbf{x}})} w_{ \pm, 0}(\overline{\mathbf{x}}(-t))
\end{aligned}
$$

With this background, we return to the discussion of the behavior of the generators $w_{ \pm}\left(\mathbf{x}^{\prime}\right)$ on the reference trajectory. For times $t<\lambda^{-1}$ (corresponding to energies $\epsilon>\hbar \lambda), \sigma_{ \pm} \approx v_{F} \lambda_{F} t \mathbb{1}$, implying that only points $\overline{\mathbf{x}}$ in the immediate vicinity of the trajectory contribute to the integral (B1). As a result, $w_{ \pm}\left(\mathbf{x}^{\prime}, t\right) \ll 1$ and the Green function remains essentially metallic. For $t>\lambda^{-1}$, the component of the matrix $\sigma_{+}$corresponding to the largest Liapunov exponent, behaves as $\left(\sigma_{+}\right)_{00} \sim v_{F} \lambda_{F} e^{\lambda t} / \lambda$, and the integral begins to sample an exponentially growing region around the reference trajectory. For

$$
\frac{v_{F} \lambda_{F}}{\lambda} e^{\lambda t} \sim a^{2} \Rightarrow t \sim t_{\mathrm{E}} \Rightarrow \epsilon \sim \hbar / t_{\mathrm{E}}
$$

field configurations $w_{+0}\left(y_{i} \sim a\right)=\mathcal{O}(1)$ contribute to the integral, which means that $w_{+}\left(\mathbf{x}^{\prime}, t>t_{\mathrm{E}}\right)=\mathcal{O}(1)$. This identifies the inverse of the Ehrenfest time as the crossover scale beyond which the Eilenberger Green function, even on exceptionally long trajectories, is locked to the superconductor order parameter.

[1] C. W. J. Beenakker, Rev. Mod. Phys. 69, 731 (1997).

[2] A. D. Zaikin, in Nonequilibrium Superconductivity, edited by V. L. Ginzburg, Nova Science Publications (1988).

[3] C. J. Lambert and R. Raimondi, J. Phys. -Condens. Matter 10, 901 (1998).

[4] P. de Gennes and D. Saint-James, Phys. Lett. 4, 151 (1963).

[5] W. McMillan, Phys. Rev. 4, 537 (1968).

[6] A. Golubov and M. Y. Kuprianov, J. Low. Temp. Phys. 70, 83 (1988).

[7] A. Golubov and M. Y. Kuprianov, Sov. Phys. JETP 69, 805 (1989).

[8] F. Zhou, B. Spivak, and A. Zyzuzin, Phys. Rev. B 52, 4467 (1995).

[9] W. Belzig, C. Bruder, and G.Schön, Phys. Rev. B 54, 9443 (1996).

[10] A. Altland, B. D. Simons, and D. Taras-Semchuk, Adv. Phys. 49, 321 (2000).

[11] A. Altland and M. R. Zirnbauer, Phys. Rev. Lett. 76, 3420 (1996).

[12] J. Melsen, P. Brouwer, K. Frahm, and C. Beenakker, Europhys. Lett. 35, 7 (1996).

[13] J. Melsen, P. Brouwer, K. Frahm, and C. Beenakker, Physica Scripta T 69, 223 (1997).
[14] P. Brouwer, Y. Oreg, B. D. Simons, and A. Altland, Phys. Rev. Lett. 82, 1269 (1999).

[15] A. F. Volkov, Phys. Rev. Lett. 74, 4730 (1995).

[16] A. A. Clerk, P. W. Brouwer, and V. Ambegaokar, Phys. Rev. B 62, 10226 (2000).

[17] A. Lodder and Y. V. Nazarov, Phys. Rev. B 9, 5783 (1998).

[18] H. Schomerus and C. W. J. Beenakker, Phys. Rev. Lett. 82, 2951 (1999).

[19] I. Kosztin, D. L. Maslov, and P. M. Goldbart, Phys. Rev. Lett. 75, 1735 (1995).

[20] P. W. Brouwer and C. W. J. Beenakker, Chaos, Solitons and Fractals 8, 1249 (1997).

[21] W. Ihra, M. Leadbeater, J. L. Vega, and K. Richter, cond-mat/9909100.

[22] K. Usadel, Phys. Rev. Lett. 25, 507 (1970).

[23] S. Pilgram, W. Belzig, and C. Bruder, Physica B 1, 442 (2000).

[24] I. L. Aleiner and A. I. Larkin, Phys. Rev. B 54, 14423 (1996).

[25] B. A. Muzykantskii and D. E. Khmelnitskii, JETP Letters 62, 76 (1995).

[26] A. V. Andreev, B. D. Simons, O. Agam, and B. L. Altshuler, Nucl. Phys. B 482, 536 (1996).

[27] A. F. Andreev, Zh. Eksp. Teor. Fiz. 46, 1823 (1965) [Sov. Phys. JETP 19, 1228 (1965)].

[28] The conversion of electrons to holes is accompanied by a phase shift of $i \exp (i \phi)$, and the reciprocal conversion of holes into electrons by $i \exp (-i \phi)$. A trajectory containing a twofold conversion, electron $\rightarrow$ hole $\rightarrow$ electron, therefore comes with a total phase shift of $i^{2}=-1=$ $\exp \left(i \hbar^{-1} h / 2\right)$.

[29] A. Golubov and M. Y. Kuprianov, JETP Lett. 61, 853 (1989).

[30] A. Altland, B. D. Simons, and D. Taras-Semchuk, Pis'ma v Zh. Eksp. Teor. Fiz. 67, 22 (1998) [JETP Lett. 67, 21 (1998)].

[31] M. G. Vavilov, P.W. Brouwer, V. Ambegaokar, and C. W. J. Beenakker, cond-mat/0006375.

[32] Notice that, in the context of superconductor problems, there is a profound difference between the trajectory oriented semiclassical approach and the quasiclassical approach. Whereas the former is based on a summation over classical trajectories of fixed energy, the latter essentially amounts to an expansion of the Gorkov equations to leading order in $\hbar$. Although both approaches use $\hbar$ as a small expansion parameter, they are fundamentally different. See Ref. [43] for a detailed discussion of this point.

[33] Under quantum dot conditions, $\epsilon<\hbar D / L^{2}$, the defining equation for the diffusion mode (diffuson), $\left(\hbar D \partial_{\mathbf{x}}^{2}+\right.$ $i \epsilon) \Pi\left(\mathbf{x}, \mathbf{x}^{\prime}\right)=\delta\left(\mathbf{x}-\mathbf{x}^{\prime}\right)$ is solved by $\Pi\left(\mathbf{x}, \mathbf{x}^{\prime}\right) \simeq(i \epsilon L)^{-1}$. (See e.g. Ref. 44.).

[34] G. Eilenberger, Z. Phys. B 214, 195 (1968).

[35] The quasiclassical Green function is related to the impurity averaged Gorkov Green function $G$ through $g(\mathbf{r}, \mathbf{n}) \approx$ $G(\mathbf{r}, \mathbf{n})$, where

$$
G(\mathbf{r}, \mathbf{n}) \equiv \frac{i}{\pi} \int d \xi \int d \mathbf{r}^{\prime} e^{i p \mathbf{n} \cdot \mathbf{r}^{\prime} / \hbar}\left\langle G\left(\mathbf{r}+\frac{\mathbf{r}^{\prime}}{2}, \mathbf{r}-\frac{\mathbf{r}^{\prime}}{2}\right)\right\rangle
$$


and $G\left(\mathbf{r}, \mathbf{r}^{\prime}\right)$ is the microscopic Gorkov Green function. Here $\xi=v_{F}\left(p-p_{F}\right)$. The origin of the only approximate equality between the quasiclassical $g(\mathbf{r}, \mathbf{n})$ and the $p$-integrated exact Wigner transform of the Gorkov Green function $G(\mathbf{r}, \mathbf{n})$ is discussed in Appendix A.

[36] A. Altland and B. D. Simons, in Supersymmetry and Trace Formulae, edited by I. V. Lerner, J. Keating, and D. E. Khmelnitskii, Plenum Publishers (1999).

[37] K. B. Efetov, Sypersymmetry in Disorder and Chaos (Cambridge University Press, Cambridge, 1997).

[38] P. M. Ostrovsky, M. A. Skvortsov and M. V. Feigelman, cond-mat/0012478.

[39] M. R. Zirnbauer, in Supersymmetry and Trace Formulae, edited by I. V. Lerner, J. Keating, and D. E. Khmelnitskii, Plenum Publishers (1999).

[40] M. C. Risken, The Fokker-Planck Equation (Springer, Berlin Heidelberg, 1996).

[41] We note that this modelling scheme is almost certainly incomplete as it suggests that a given realization of a chaotic system auto-regularizes itself. In contrast, other approaches to the problem (cf. e.g. Ref. 39 ) indicate that some minimal averaging over a stochastic set of parameters is needed to stabilize the very formulation of a quasiclassical Green function approach.

[42] At this stage the derivation of the clean $\sigma$-model becomes problematic: There is no reason for assuming that the relevant fluctuations around the mean field are smooth. In fact, the singular phase-space structure of the eigenfunctions of the Liouville operator suggests that the opposite is the case. That we are nevertheless permitted to make a smooth ansatz has to do with the fact that the subsequent disorder average will project out all field configurations of discontinuous type. Notice that the problem outlined above equally affects the pure quasiclassical formalism where identical construction steps are performed in a non-functional integral context.

[43] J. Rammer and H. Smith, Rev. Mod. Phys. 58, 323 (1986).

[44] B. L. Altshuler and B. I. Shklovskii, Zh. Eksp. Teor. Fiz. 91, 220 (1986) [Sov. Phys. JETP 64, 127 (1986)]. 